\title{
Data-driven Covariate Selection for Confounding Adjustment by Focusing on the Stability of the Effect Estimator
}

\author{
Wen Wei Loh ${ }^{1,2}$ and Dongning Ren ${ }^{3}$ \\ ${ }^{1}$ Department of Quantitative Theory and Methods \\ Emory University, Georgia, USA \\ ${ }^{2}$ Department of Data Analysis \\ Ghent University, Gent, Belgium \\ ${ }^{3}$ Department of Social Psychology \\ Tilburg University, Tilburg, The Netherlands
}

\begin{abstract}
Author Note
Wen Wei Loh (iD https://orcid.org/0000-0001-7086-7281

Dongning Ren (iD https://orcid.org/0000-0001-7749-2419
\end{abstract}

This manuscript has been accepted for publication in Psychological Methods. It is posted online ahead of print.

WWL was supported by the Special Research Fund (BOF) of Ghent University postdoctoral fellowship BOF.PDO.2020.0045.01. The computational resources (Stevin Supercomputer Infrastructure) and services used in this work were provided by the VSC (Flemish Supercomputer Center), funded by Ghent University, FWO and the Flemish Government - department EWI. 


\begin{abstract}
Valid inference of cause-and-effect relations in observational studies necessitates adjusting for common causes of the focal predictor (i.e., treatment) and the outcome. When such common causes, henceforth termed confounders, remain unadjusted for, they generate spurious correlations that lead to biased causal effect estimates. But routine adjustment for all available covariates, when only a subset are truly confounders, is known to yield potentially inefficient and unstable estimators. In this article, we introduce a data-driven confounder selection strategy that focuses on stable estimation of the treatment effect. The approach exploits the causal knowledge that after adjusting for confounders to eliminate all confounding biases, adding any remaining non-confounding covariates associated with only treatment or outcome, but not both, should not systematically change the effect estimator. The strategy proceeds in two steps. First, we prioritize covariates for adjustment by probing how strongly each covariate is associated with treatment and outcome. Next, we gauge the stability of the effect estimator by evaluating its trajectory adjusting for different covariate subsets. The smallest subset that yields a stable effect estimate is then selected. Thus, the strategy offers direct insight into the (in)sensitivity of the effect estimator to the chosen covariates for adjustment. The ability to correctly select confounders and yield valid causal inferences following data-driven covariate selection is evaluated empirically using extensive simulation studies. Furthermore, we compare the introduced method empirically with routine variable selection methods. Finally, we demonstrate the procedure using two publicly available real-world datasets. A step-by-step practical guide with user-friendly $\mathrm{R}$ functions is included.
\end{abstract}

Keywords: Causal inference; Double selection; Observational studies; Propensity scores; Strong ignorability 


\section{Data-driven Covariate Selection for Confounding Adjustment by Focusing on the Stability of the Effect Estimator}

\section{Introduction}

In observational studies with non-randomized treatments, causal inference requires adjusting for confounders, i.e., common causes of the treatment and outcome. Unbiased estimation of a causal effect thus assumes that adjusting for a set of measured covariates suffices to eliminate all confounding (often termed strong ignorability; Rosenbaum and Rubin, 1983). To justify this assumption, researchers must use theoretical and conceptual knowledge to compile and record a comprehensive list of covariates (Pearl, 2009, Rubin, 2007; Steiner et al., 2010) 1

But routinely adjusting for all available covariates may be undesirable. An extensive list of covariates may contain non-confounders, i.e., those associated with only treatment or outcome but not both. Under such scenarios, adjusting (or statistically controlling) for all covariates - including non-confounders - has several drawbacks. First, it can lead to an unnecessary loss of statistical power or induce finite-sample bias even if strong ignorability is satisfied. For example, adjusting for non-confounding but strong predictors of treatment does not reduce confounding bias but can potentially produce highly imprecise estimators (Austin et al., 2007; Brookhart et al., 2006; VanderWeele, 2019). Second, including extraneous non-confounding predictors raises the risks of biases due to incorrectly specified regression models (Drake, 1993; Vansteelandt et al., 2012). Third, striving to eliminate systematic imbalances in the distributions of non-confounding predictors across different treatment groups can create an undue burden on researchers (Kainz et al., 2017) and potentially detract from checking covariate balance and overlap diagnostics for the true confounders.

\footnotetext{
1 The comprehensive list of covariates should be measured prior to treatment (Gische et al., 2021; Little et al., 2000, Rosenbaum, 1984, 2002, Shadish \& Steiner, 2010), and should not include variables that induce "collider (stratification) bias" (Elwert \& Winship, 2014 Thoemmes, 2015).
} 
An approach to address these challenges is to use data-driven (or data-adaptive) covariate or confounder selection methods (Witte \& Didelez, 2019). Starting with a rich set of putative confounders that are assumed to satisfy strong ignorability, these methods empirically screen for non-confounders and single out a covariate subset that remains sufficient to eliminate confounding biases. Such data-driven methods supplement substantive theory-based covariate selection to improve causal effect estimation. Although these methods are routinely adopted in other fields, such as medicine and epidemiology (VanderWeele, 2019; Witte \& Didelez, 2019), the recent advances have hitherto been absent in psychological research.

In this article, we introduce a state-of-the-art data-driven confounder selection strategy. This strategy builds upon recent work in causal inference for medical and health services research (Loh \& Vansteelandt, 2021a). We present this strategy because, as we will elaborate on later, it offers a systematic and principled approach to guide a data-driven covariate selection process. This strategy also has several appealing features that are particularly pertinent to psychological researchers. In the following sections, we describe the proposed strategy, present a comprehensive simulation study comparing the proposal with existing routine variable selection methods, illustrate the proposal using two publicly available real-world datasets, and conclude with a discussion section. All R (R Core Team, 2021) scripts used to implement the proposed strategy and the methods under comparison, in the simulation study and applied examples, are freely available online. $2^{2}$

\section{Data-driven confounder selection focusing on the stability of the effect estimator}

The proposed data-driven confounder selection procedure proceeds in two steps.

Step 1. Order the covariates in decreasing priority for confounding adjustment.

Step 2. Calculate the treatment effect estimator given each nested covariate subset indexed

2 https://github.com/wwloh/covariate-selection-effect-stability 
by the ordered covariates. Select the smallest subset that yields the most stable effect estimate.

In what follows, we illustrate each step using a simple example with three covariates $\left(L_{1}, L_{2}, L_{3}\right)$, a binary treatment $(A)$, and a continuous outcome $(Y)$. For ease of exposition here, we focus our presentation on linear logistic regression models for the treatment and linear regression models for the outcome, with no missing data. Possible extensions that permit non-linear treatment and outcome models, and account for missing data, are described in the Discussion section.

\section{Step 1. Prioritizing covariates for confounding adjustment}

In the first step, the covariates are hierarchically ordered to reflect their decreasing priority for confounding adjustment. Therefore, the ordered covariates index a series of nested covariate subsets: the smallest non-empty subset contains the most important covariate, the second smallest subset includes the two most important covariates, and so on, with the largest subset containing all the covariates. Let $\mathcal{L}^{j}$ denote the nested subsets containing $j$ covariates for $j=0, \ldots, J$, where $J$ is the number of measured covariates and $\mathcal{L}^{0}$ contains no covariates. We illustrate the procedure using the simple example (with $J=3)$.

A1. (a) Fit a treatment model by regressing treatment on each covariate separately. For example, a logistic regression model for the binary treatment would be:

$$
\operatorname{logit}\left\{\mathrm{E}\left(A \mid L_{k}\right)\right\}=\alpha_{0,0}+\alpha_{0, k} L_{k} ; k=1,2,3,
$$

where $\operatorname{logit}(x)=\log \{x /(1-x)\}$ is the logit link function $3^{3}$

(b) Similarly, fit an outcome model by regressing the outcome on the treatment and each of the covariates separately. For example, a linear regression model for the

\footnotetext{
${ }^{3}$ For a binary treatment, the conditional probability that an individual would have received or selected treatment given a set of measured covariates is commonly termed the propensity score (Rosenbaum \& Rubin, 1983).
} 
continuous outcome would be:

$$
\mathrm{E}\left(Y \mid A, L_{k}\right)=\beta_{0,0}+\beta_{0, A} A+\beta_{0, k} L_{k} ; k=1,2,3 .
$$

A2. Among the three candidates, add the covariate that is most strongly associated with either treatment $A$ or outcome $Y$ to the subset $\mathcal{L}^{1}$. For example, if $\alpha_{0,2}$ is the coefficient with the smallest p-value among the six coefficients $\left(\alpha_{0, k}, \beta_{0, k}\right), k=1,2,3$, then $\mathcal{L}^{1}=\left\{L_{2}\right\}$. In other words, the covariate with the smallest p-value - across both treatment and outcome models - is considered the most important covariate for confounding adjustment, according to this specified criterion.

A3. (a) Fit a treatment model by regressing treatment on the covariate in $\mathcal{L}^{1}$, and each of the remaining covariates. For example:

$$
\operatorname{logit}\left\{\mathrm{E}\left(A \mid \mathcal{L}^{1}, L_{k}\right)\right\}=\alpha_{1,0}+\alpha_{1,2} L_{2}+\alpha_{1, k} L_{k} ; k=1,3 .
$$

(b) Similarly, fit an outcome model by regressing the outcome on the treatment, the covariate in $\mathcal{L}^{1}$, and each of the remaining covariates. For example:

$$
\mathrm{E}\left(Y \mid A, \mathcal{L}^{1}, L_{k}\right)=\beta_{1,0}+\beta_{1, A} A+\beta_{1,2} L_{2}+\beta_{1, k} L_{k} ; k=1,3 .
$$

A4. Among the two remaining candidates, add the covariate that is most strongly associated with either treatment $A$ or outcome $Y$ to the subset $\mathcal{L}^{2}$. For example, if $\beta_{1,3}$ is the coefficient with the smallest p-value among the four coefficients $\left(\alpha_{1, k}, \beta_{1, k}\right), k=1,3$, then $\mathcal{L}^{2}=\left\{L_{2}, L_{3}\right\}$. We reiterate that the covariate with the smallest p-value - across both treatment and outcome models - is considered the more important covariate for confounding adjustment, according to this specified criterion.

A5. Add the remaining covariate to the subset $\mathcal{L}^{3}$. For example, $\mathcal{L}^{3}=\left\{L_{2}, L_{3}, L_{1}\right\}$.

Carrying out the steps above returns a hierarchical ordering of the covariates based on their decreasing priority for confounding adjustment (according to the specified criterion). 
For example, $L_{2}$ is the most important covariate for confounding adjustment, $L_{3}$ is the next most important, and $L_{1}$ is the least important. Therefore, the ordered covariates index a series of nested subsets, $\mathcal{L}^{1} \supset \mathcal{L}^{2} \supset \mathcal{L}^{3}$.

A feature of the proposal is thus explicating the (hierarchical) order in which covariates are systematically adjusted for. Researchers can further refine the priorities by synthesizing theoretical knowledge, external empirical evidence, or practical considerations. For example, the most crucial covariates from various heterogeneous domains (Steiner et al., 2015: Steiner et al., 2010) can be assigned the highest priorities so that all larger subsets will contain these covariates. Furthermore, the series of nested covariate subsets indexed by the prioritized covariates reinforce current recommendations to build a pool of different covariate subsets for confounding adjustment (Kainz et al., 2017; Keele \& Small, 2021).

\section{Criterion for prioritizing covariates}

The criterion used to prioritize covariates in steps A2 and A4 is based on simultaneously assessing the (partial) associations between treatment and the covariates, and between outcome and the covariates. Such an approach is inspired by "double selection" (Belloni et al., 2014) principles. Double selection exploits empirical information on both the covariate-treatment and covariate-outcome associations simultaneously toward increasing the chances of selecting true confounders. In contrast, limiting assessment to covariate-treatment (or covariate-outcome) associations only entirely ignores the association of each candidate covariate with the outcome (or treatment) $4^{4}$ Under the proposed strategy, discarding such information can only lower the priorities for confounders more strongly associated with one than the other, potentially raising the risk of bias from omitting measured confounders.

\footnotetext{
4 For example, traditional "design of observational studies" advocates considering only covariate-treatment relations for confounding adjustment, without access to or knowledge of the observed outcomes (Rubin, 2007; Stuart \& Rubin, 2008).
} 
We emphasize that the p-values in steps A2 and A4 are used only to quantify the strengths of association between each candidate covariate with treatment and with outcome - relative to other candidates - using a common scale and not for inference on the causal effect directly. In principle, any criterion that permits ordering or ranking the covariates can be readily used to prioritize covariates instead. In the Discussion section, we describe other possibilities.

The nested subsets, e.g., $\mathcal{L}^{1} \supset \mathcal{L}^{2} \supset \mathcal{L}^{3}$, constructed in this first step of the procedure may prima facie resemble a forward selection procedure for adding predictors to a regression model. But there are four fundamental distinctions. First, a forward selection procedure is limited to a single regression model. In contrast, we consider both the treatment model and the outcome model simultaneously. Second, forward selection procedures stop adding predictors based solely on a predetermined significance level; e.g., once the model fit does not statistically significantly improve beyond a certain level. In contrast, we emphasize that no covariates are precluded from confounding adjustment following this first step of the procedure, because no predetermined (significance-based) threshold is imposed to rule out covariates based solely on the absence of association. Third, as we will explain in the next section, we build on the established "change-in-estimate" approach for covariate selection in epidemiological research (Greenland et al., 2016; Maldonado \& Greenland, 1993 Mickey \& Greenland, 1989) to directly evaluate the impact of adjusting for each additional covariate on the quality of the treatment effect estimator. Finally, the goals of (data-driven) covariate selection for causal inference differ substantively from routine variable selection methods: the former seeks confounders associated with both treatment and outcome, whereas the latter seeks pure predictors of either treatment or outcome based on a single regression model. We refer interested readers to Greenland (2008), VanderWeele (2019), and Witte and Didelez (2019) for more in-depth discussions. 


\section{Step 2: Assessing the stability of the effect estimator}

In the second step, we evaluate the trajectory of the treatment effect estimator across the nested covariate subsets indexed by the prioritized covariates in the first step. We briefly introduce the notation for presenting the effect estimator for evaluating stability. Let $Y^{a}$ denote an individual's potential outcome for $Y$ if the individual had been assigned to treatment level $A=a$, possibly counter to actuality. $\left.\right|^{5}$ For a binary treatment $A$, we define $\psi=\mathrm{E}\left(Y^{1}\right)-\mathrm{E}\left(Y^{0}\right)$ as the marginal or average causal effect comparing treated $(a=1)$ and untreated $(a=0)$ individuals ${ }^{6}$

B1. Calculate a treatment effect estimator $\widehat{\psi}_{j}$ given the covariates selected in each subset $\mathcal{L}^{j}$, for $j=0, \ldots, J$.

B2. Gauge the stability of the bias of each estimator $\left(\widehat{\psi}_{j}-\psi\right)$ in the sequence indexed by the nested covariate subsets.

B3. Select the smallest covariate subset that yields a relatively stable value of the bias after considering sampling variability.

We illustrate each step using the above simple example.

\section{Step B1}

Continuing our example, $\widehat{\psi}_{0}$ is the effect estimator without adjusting for any covariates, $\widehat{\psi}_{1}$ is the effect estimator adjusting for the most important covariate $\left(L_{2}\right)$ in $\mathcal{L}^{1}$,

${ }^{5}$ Under the Neyman-Rubin potential outcomes framework (Rubin, 1990 Splawa-Neyman et al., 1990), a (binary) treatment must be potentially manipulable, and it must be conceptually conceivable for each individual in the population of interest to be either treated or untreated so that both of their potential outcomes are well-defined (Holland, 1986; Rubin, 1986).

${ }^{6}$ In this paper, we present the proposed strategy using a binary treatment for simplicity. In principle, the proposal can be readily extended to quantitative or continuous-valued treatments by utilizing generalized propensity scores for the treatment (Austin, 2018 Hirano \& Imbens, 2004). Such extensions are beyond the scope of this paper and deferred to future work. 
$\widehat{\psi}_{2}$ is the effect estimator adjusting for the two most important covariates $\left(L_{2}\right.$ and $\left.L_{3}\right)$ in $\mathcal{L}^{2}$, and $\widehat{\psi}_{3}$ is the effect estimator adjusting for all $J=3$ covariates. The nested covariate subsets therefore index a sequence of effect estimators; e.g., $\left\{\widehat{\psi}_{0}, \widehat{\psi}_{1}, \widehat{\psi}_{2}, \widehat{\psi}_{3}\right\}$. We describe how to calculate an effect estimator in the next section.

\section{Step B2}

When there exists a subset of the measured covariates that suffices to eliminate all confounding, as would be possible under strong ignorability, further adjusting for other covariates that are unrelated to either treatment or outcome, or both, should not systematically alter the treatment effect estimator. To see why suppose that $L_{2}$ was the only common cause of treatment $A$ and outcome $Y$ in the true data-generating process so that strong ignorability holds given $L_{2}$. For simplicity, suppose that the treatment model given $L_{2}$ and the outcome model given $A$ and $L_{2}$ are correctly specified, and that the sample size is sufficiently large as to ignore sampling variability. Then the effect estimator $\widehat{\psi}_{1}$ that adjusts for $L_{2}$ - in the smallest non-empty covariate subset $\mathcal{L}^{1}$ - would be unbiased. Further adjusting for the extraneous covariates $\left(L_{1}\right.$ and $\left.L_{3}\right)$ that are non-confounders of $A$ and $Y$ should not systematically shift the estimates of $\psi$. Hence, we could conclude that the smallest subset $\mathcal{L}^{1}$ (with only $L_{2}$ ) yielding an unaltered estimator, even as more covariates are added, suffices to eliminate confounding bias.

We exploit this causal knowledge to select the smallest subset for confounding adjustment. Because our interest is in eliminating confounding bias, ideally we seek to evaluate the stability of the bias of each estimator $\left(\widehat{\psi}_{j}-\psi\right)$ across the nested covariate subsets. But there are three practical challenges to comparing the biases of the estimators across different subsets. First, the true value of $\psi$ is unknown. Second, the sampling uncertainty of the estimators have to be accounted for. Third, the estimators are correlated because they use the same empirical data. To overcome these challenges, we propose using the standardized difference between the estimator in each subset and the "benchmark" estimator $\widehat{\psi}_{J}$ that adjusts for all available covariates (i.e., from the largest subset). The 
standardized difference can be defined as:

$$
\frac{\widehat{\psi}_{j}-\widehat{\psi}_{J}}{\sqrt{\operatorname{var}\left(\hat{\psi}_{j}-\widehat{\psi}_{J}\right)}}, \quad j=0, \ldots, J-1 ;
$$

where $\operatorname{var}(X)$ denotes the asymptotic variance of $X$. The choice of $\widehat{\psi}_{J}$ as a benchmark is merely motivated by its unbiasedness in very large samples under strong ignorability, so

that the difference $\widehat{\psi}_{j}-\widehat{\psi}_{J}$ can be viewed as an approximate bias of $\widehat{\psi}_{j}$ relative to the true value of $\psi$. In general, other benchmarks can be readily considered but are beyond the scope of this paper. The sampling variability of the effect estimators are accounted for (in the denominator) so that the values of (1) across different subsets are on the same scale. Correlations between different subsets can be taken into account through their shared dependence on the common benchmark estimator.

\section{Step B3}

The smallest subset that yields the most stable value of (1) can then be selected. For example, the values of (1) may be plotted for each subset, as we will illustrate using the applied example later. The smallest subset that yields a value that does not "fluctuate" as the subsets enlarge can then be selected through visual inspection. Alternatively, a diagnostic that numerically assesses the relative stability or (in)sensitivity of the difference $\widehat{\psi}_{j}-\widehat{\psi}_{J}$, while taking into account its sampling variability, can directly optimize the quality of the treatment effect estimator. We describe how to calculate such a diagnostic as defined in $\mathrm{A} 2$ of Appendix $\mathrm{A}$

\section{Treatment effect estimator}

A feature of the proposal is that any estimator of the marginal treatment effect $\psi$ can be used depending on researchers' modeling preferences. Because both treatment and outcome models are used to prioritize the covariates in the first step, we propose an estimator that leverages both models in the second step. In this paper, we recommend the doubly-robust augmented inverse probability of treatment weighted (DR-AIPW) estimator (Lunceford \& Davidian, 2004; Robins et al., 1994). The DR-AIPW estimator combines 
regression adjustment in an outcome model with the inverse probabilities of receiving treatment based on a treatment model. The outcome model exploits information about the treatment effect to predict potential outcomes, without necessarily parametrizing the marginal treatment effect $\psi$ as a regression coefficient. The inverse probabilities of receiving treatment (given a set of observed covariates) create a pseudo-population where the different treatment groups have the same weighted distribution on the set of covariates. Under strong ignorability and a correctly specified model for treatment given the covariates, treatment is no longer associated with the covariates in the pseudo-population, and confounding is thus removed. We present the DR-AIPW estimator and describe a closed-form expression for (1) in Appendix B.

The class of DR-AIPW estimators is endowed with several attractive characteristics (Glynn \& Quinn, 2010). First, they are asymptotically unbiased when both the treatment model and outcome model are correctly specified and consistent if only one model is correctly specified (Bang \& Robins, 2005, Kang \& Schafer, 2007). Second, they can mitigate the risks of biases due to misspecified models by using richly parameterized regression models with flexible functional forms for the predictors (Glynn \& Quinn, 2010; Kang \& Schafer, 2007), or nonparametric machine learning algorithms (Zhong et al., 2021), for both treatment and outcome models to construct the estimator. Third, in contrast to an estimator that uses the treatment model only, a correctly specified outcome model in the DR-AIPW estimator exploits information about the treatment effect and stabilizes the estimator when the (estimated) probabilities of treatment are close to zero or one result in volatile weights for some observations (Glynn \& Quinn, 2010). . $^{7}$ Finally, in contrast to an estimator that uses the outcome model only, a correctly specified treatment model in the

7 Under certain data-generating scenarios, a doubly-robust estimator with misspecified treatment and outcome models can be empirically more biased than an estimator that only uses a misspecified treatment model (Kang \& Schafer, 2007). But a doubly-robust estimator offers protection against a misspecified treatment model when the outcome model is correctly specified, whereas an estimator that relies on only a treatment model remains at risk of biases due to a misspecified treatment model. 
DR-AIPW estimator can protect the estimator from biases due to extrapolation in the outcome model when confounders strongly associated with both treatment and outcome result in reduced covariate overlap (Vansteelandt \& Daniel, 2014).

\section{Simulation study}

We conducted a simulation study using various data-generating scenarios to empirically evaluate the ability of the data-driven covariate selection strategy to: (i) correctly select confounders, (ii) yield unbiased estimators, and (iii) return null hypothesis tests that preserved the nominal type I error rate.

\section{Data-generating process}

We will start with one scenario and defer details of other scenarios to a later section. In a given scenario, the $J$ observed covariates were partitioned into four subsets as follows. Confounders in the first subset simultaneously affected treatment and outcome; their indices were denoted by $\mathcal{S}_{1}=\{1,2\}$. Covariates in the second subset affected outcome only; their indices were denoted by $\mathcal{S}_{2}=\{3,4\}$. So-called "instruments" (Brookhart et al., 2006: Pearl, 2011; Wooldridge, 2016) in the third subset were associated with treatment only; their indices were denoted by $\mathcal{S}_{3}=\{5,6\}$. Covariates in the fourth subset were unassociated with either treatment or outcome; their indices were denoted by $\mathcal{S}_{4}=\{7, \ldots, J\}$. Hence the assumption that strong ignorability holds given all $J$ covariates is still satisfied.

Datasets with sample size $n=400$ and $J=20$ covariates were then generated under the null of no treatment effect $(\psi=0)$ as follows. Let $L_{i s}$ denote the $s$-th observed covariate for individual $i$. For $s=1, \ldots, J$, each covariate was randomly (and independently) drawn from a standard normal distribution; i.e., $L_{i s} \sim \mathcal{N}(0,1)$. Denote the resulting vector of $J$ covariates by $L_{i}=\left(L_{i 1}, \ldots, L_{i J}\right)$. The underlying treatment was determined as $A_{i}^{*}=\sum_{s=1}^{J} \gamma_{s} L_{i s}$, where $\gamma_{s}=1.0$ if $s \in \mathcal{S}_{1}$ (a confounder), or $\gamma_{s}=r$ if $s \in \mathcal{S}_{3}$ (an instrument), or 0.0 otherwise. We set $r=0.5$ so that the instrument-treatment associations were weaker than the confounder-treatment associations, and to avoid 
inducing perfect separation in the treatment. The observed treatment was then randomly drawn as $A_{i} \sim \operatorname{Bernoulli}\left\{\operatorname{expit}\left(A_{i}^{*}\right)\right\}$, where $\operatorname{expit}(x)=\exp (x) /\{1+\exp (x)\}$. The underlying outcome was determined as $Y_{i}^{*}=\sum_{s=1}^{J} \beta_{s} L_{i s}$, where $\beta_{s}=1.0$ if $s \in \mathcal{S}_{1}$ (a confounder), or $\beta_{s}=z$ if $s \in \mathcal{S}_{2}$ (an outcome-only predictor), or 0.0 otherwise. We set $z=1.0$ so that the predictors of outcome only and the confounders each had the same strength of association with the outcome. The observed outcome was then randomly drawn as $Y_{i} \sim \mathcal{N}\left(Y_{i}^{*}, \sigma^{2}\right)$, where $\sigma=\max _{i}\left|Y_{i}^{*}\right|$.

\section{Variable selection methods under comparison}

We compared the ability of the proposal to correctly select the true confounders against routine variable selection methods for linear regression models and structural equation models (SEM) implemented in publicly available R (R Core Team, 2021) packages. We applied each variable selection method to the outcome model following existing recommendations to adjust for covariates associated with the outcome (confounders or not), and to avoid including pure predictors of treatment; see, e.g., Austin et al. (2007) and Brookhart et al. (2006). Because our focus was on covariate selection, we considered a correctly specified linear regression model for outcome with main effects for treatment and all the covariates. We briefly describe each variable selection method (labelled with the prefix "S") and refer readers to the respective references for further details.

S1. The proposed method as described in the preceding section. We used a correctly specified logistic regression model for treatment with main effects for the covariates to construct the DR-AIPW estimator. The subset with the most stable effect estimator was selected using the numerical diagnostic $\mathrm{A} 2 \mathrm{C}$ described in Appendix A

S2. Regularized (or penalized) linear regression using the least absolute shrinkage and selection operator (LASSO; Tibshirani, 2011). The optimal value of the LASSO penalty (that minimized the mean squared error) in a regression model was selected using cross-validation with the cv.gImnet function (Friedman et al., 2010). Because 
our interest was in the treatment effect on the outcome, prematurely shrinking the coefficient of treatment to zero can lead to biased inference. The coefficient for treatment was therefore better left unpenalized, e.g., by setting its penalty factor to zero, to retain treatment in the outcome model. All other options were set to their default levels. Covariates with non-zero coefficient estimates at the optimal penalty value were deemed to have been selected.

S3. Regularized SEM that extends regularization for single regression models to joint models (RegSEM; Jacobucci et al., 2016). In each model, the (single) optimal value of the LASSO penalty was selected from among 100 possible values using cross-validation with the cv_regsem function (Jacobucci et al., 2020). All other options were set to their default values. The coefficient for treatment was similarly left unpenalized to retain treatment in the outcome model, while all other coefficients could be penalized. Covariates with non-zero coefficient estimates at the optimal penalty value were deemed to have been selected.

S4. Semi-confirmatory SEM ("SC-SEM") via penalized likelihood that extends penalization for single regression models to joint models (Huang et al., 2017). We employed the methods implemented in the Islx package (Huang, 2020a). A model was fitted by optimizing a penalized likelihood loss function, given a fixed value of the LASSO penalty. We set the argument start_method="heuristic" and all other options to their default values. The coefficient for treatment was similarly left unpenalized to retain treatment in the outcome model, while all other coefficients could be penalized. The same specified model was fitted under each of a considered set of penalty values, and the optimal value selected using the Adjusted Bayesian Information Criterion (ABIC; Sclove, 1987) following the online package vignette 8 The penalized coefficient estimates under the optimal penalty value were then

8 https://cran.r-project.org/web/packages/lslx/vignettes/structural-equation-modeling.html 
extracted, and covariates with non-zero estimates deemed to have been selected.

\section{Results for covariate selection}

The ability to correctly select confounders using either the proposal (S1) or routine variable selection methods (S2 - S4) were evaluated empirically using 1000 simulated datasets. We plotted the empirical probability that exactly both true confounders were selected (vertical axis) against the proportion of available covariates selected (horizontal axis) for each method in Figure 1. The proposed stability-based method was far more likely to select both confounders, albeit selecting larger adjustment sets, than applying routine variable selection methods to the outcome model. 


\section{Figure 1}

Scatterplot of the empirical average probabilities of selecting exactly both true confounders (vertical axis) against the empirical average proportion of available covariates selected (horizontal axis), for each covariate or variable selection method.
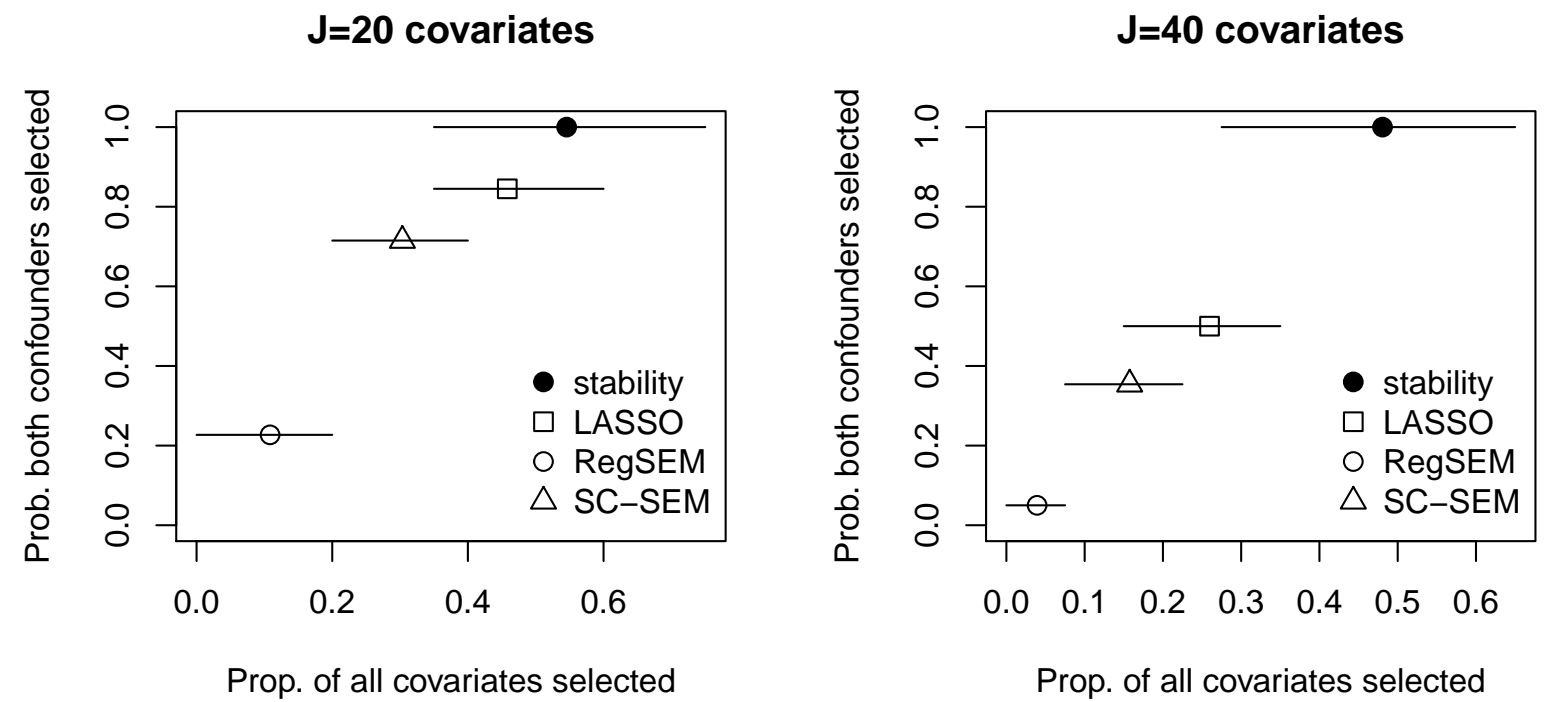

Note. In the left panel, there were $J=20$ covariates and the regression coefficients for the predictors of outcome only were $z=1.0$; in the right panel, there were $J=40$ covariates and the regression coefficients for the predictors of outcome only were $z=1.5$. Each horizontal bar represents the interquartile range of the proportion of available covariates selected (over all simulated datasets). 


\section{Effect estimators (following variable selection) under comparison}

Next, we assessed the ability of the covariates selected using each method ( $\mathrm{S} 1-\mathrm{S} 4)$ to yield unbiased statistical inference. For each method, we refitted the treatment (outcome) model with main effects for (treatment and) only the selected covariates, then used the refitted regression models to calculate the DR-AIPW estimator.

We also compared the above estimators using the selected covariates against estimators using known covariate subsets (labelled with the prefix "M"). Given each of the following covariate subsets, the DR-AIPW estimator was calculated using a treatment model with main effects for the covariates, and an outcome model with main effects for treatment and the covariates:

M1. No covariates.

M2. Predictors of treatment only (instruments; $L_{i s}, s \in \mathcal{S}_{3}$ ).

M3. The true confounders $\left(L_{i s}, s \in \mathcal{S}_{1}\right)$, and the outcome-only predictors $\left(L_{i s}, s \in \mathcal{S}_{2}\right)$.

M4. The true confounders $\left(L_{i s}, s \in \mathcal{S}_{1}\right)$, and the treatment-only predictors $\left(L_{i s}, s \in \mathcal{S}_{3}\right) .9$

We used M1 to demonstrate the biases due to omitted confounders, and M2 to demonstrate the undesirable amplification of confounding bias that can arise when true confounders are unadjusted for, but pure predictors of treatment only are adjusted for (Brookhart et al., 2006; Pearl, 2011; Wooldridge, 2016). The "target" set of covariates in M3 included all predictors of outcome - regardless of their relationship with treatment and excluded all predictors (of treatment only) that were unrelated to outcome. The set of covariates in M4 included all predictors of treatment, even pure predictors of treatment that were unrelated to outcome. For all the above methods, in addition to the DR-AIPW estimator, we also calculated its standard error and the p-value from a test of the null hypothesis of zero effect.

\footnotetext{
${ }^{9}$ We thank a reviewer for suggesting this benchmark model M4 for comparison.
} 
We further considered two prevalent methods which utilize a treatment model only (i.e., without an outcome model). Both methods leverage machine learning algorithms to directly optimize covariate balance when fitting a model for treatment given all available covariates ${ }^{10}$ In doing so, the covariates whose distributions must be balanced are implicitly selected, potentially doing away with the need for separate data-driven covariate selection. We used the estimated probabilities of treatment from either method to calculate an inverse probability of treatment weighted (IPW) estimator (Austin \& Stuart, 2015; Lunceford \& Davidian, 2004) of the treatment effect $\psi$. Similar to the DR-AIPW estimator, the weights create a pseudo-population without systematic differences in the covariate distributions between the different treatment groups. We briefly describe each method and refer readers to the respective references for further details.

M5. Covariate balancing propensity scores (CBPS; Imai and Ratkovic, 2014) as implemented in the eponymous function and package (Fong et al., 2021). CBPS uses a parametric logistic regression model as the treatment model. But instead of maximizing the likelihood and model fit to determine the model coefficient values, CBPS directly optimizes the balance of the covariate distributions across different treatment groups by solving estimating functions that impose balance conditions on the covariates ${ }^{11}$ For simplicity, we assumed a correctly-specified treatment model with main effects for all the covariates. We set the options ATT $=0$ to estimate the average treatment effect, and iterations $=10000$ to increase the default number of iterations ten-fold; all other options were set at their default values.

${ }^{10}$ A key property of the propensity score is that the observed covariate distributions are rendered (approximately) similar, or "balanced," across the treated and untreated groups by conditioning on the propensity score, provided strong ignorability holds, and the model for the treatment given the covariates is correctly specified (Rosenbaum \& Rubin, 1983).

${ }^{11}$ A logistic regression model fitted with standard maximum likelihood can be sensitive to outliers (Pregibon, 1982), and maximizes predictive accuracy which is neither necessary nor sufficient to eliminate observed confounding bias (Westreich et al., 2011). 
M6. Generalized boosted models (GBM; McCaffrey et al., 2004) as implemented in the ps function of the twang package (Cefalu et al., 2021). GBM exploits a machine learning technique called gradient boosting to maximize covariate balance without assuming a parametric treatment model that can be vulnerable to model misspecification bias. We considered GBM because it is an attractive nonparametric alternative to CBPS under certain settings (Setodji et al., 2017). We used as a stopping rule for the optimization algorithm the maximum of the "standardized effect size (or bias)," defined as the (weighted) difference in covariate means between the treated and untreated groups, divided by the pooled (weighted) standard deviation, using the same (inverse probability of treatment) weights for calculating the effect estimator (stop. method $=$ 'es.max'). All other options were set at their default values.

\section{Results for effect estimation}

The resulting empirical biases and type I error rates of the DR-AIPW effect estimator adjusting for either selected (S1 - S4) or known (M1 - M4) covariates are displayed in Table 1. The stability method (S1) - that selected both confounders with high empirical probability - yielded unbiased point estimates and hypothesis tests with type I error rates at the nominal level empirically. The routine variable selection methods (S2-S4), which were empirically less likely to select both confounders, therefore led to confounding biases in the treatment effect estimates and hypothesis tests.

As expected, failing to adjust for any covariates (M1) induced unmeasured confounding of the treatment-outcome relation; moreover, adjusting for pure predictors of treatment only (M2) exacerbated the confounding bias. ${ }^{12}$ Using the target set of covariates (M3) yielded unbiased estimates and controlled the type I error rate at its nominal level empirically. We can also see that the variability induced by data-driven covariate selection

\footnotetext{
12 The worsening of unmeasured confounding bias by adjusting for instruments is termed "bias amplification" (Pearl, 2011; Wooldridge, 2016), and offers another perspective for the case against adjusting for pure predictors of treatment (VanderWeele, 2019, Westreich et al., 2011).
} 
(S1) is reflected in the higher root mean squared error (RMSE) relative to that of the target model (M3). Controlling for all predictors of treatment (M4) also yielded unbiased estimates. But omitting non-confounding predictors of outcome led to much less precise estimates (as reflected in the higher RMSE) compared to the target model (M3); moreover, the empirical RMSE was almost as high as that following data-driven covariate selection (S1). Finally, both IPW estimators using either CBPS (M5) or GBM (M6), which adjusted for all available covariates, were biased empirically; they also had higher RMSE than adjusting for confounders and pure predictors of outcome (M3) 13

\section{Other scenarios}

The simulation study was repeated under different data-generating scenarios with either: (i) more covariates, e.g., $J \in\{20,40\}$; or (ii) a stronger effect of each instrument on treatment, e.g., $r \in\{0.5,1.0,1.5\}$; or (iii) a mixture of normal and log-normal covariates; or (iv) a stronger effect of each outcome-only predictor on outcome, e.g., $z \in\{1.0,1.5\}$.

We summarize the findings across all scenarios here, and defer the detailed results for each of the 24 unique data-generating scenarios to the Supplementary Online Materials. First, the proposed stability method selected exactly both confounders at least $95 \%$ of the time empirically across all considered scenarios. Using LASSO (S2) led to both confounders being selected between $16 \%$ and $89 \%$ of the time empirically across all scenarios. Using the SEM-based methods RegSEM (S3) and SC-SEM (S4) led to confounders being selected much less frequently empirically: respectively, $30 \%$, and $79 \%$ of the time empirically at best for a specific scenario. This is because routine variable

\footnotetext{
13 To address the possibility that the empirical biases could have potentially resulted from a lack of covariate balance, we recorded the maximum standardized bias across the covariates adjusted for as a summary measure of covariate balance using the cobalt package (Greifer, 2021). Smaller values indicate better balance. The mean and maximum among all simulated datasets of this particular covariate balance measure are displayed in the two rightmost columns of Table 1 . The IPW estimators utilizing either CBPS or GBM had standardized biases no greater than those of the target estimator (M3), but nonetheless yielded biased estimates and hypothesis tests.
} 


\section{Table 1}

Empirical summaries of estimates of the treatment effect and its standard error ("SE") either following the use of each covariate or variable selection method (S1 - S4), or given known covariates (M1 - M4), or using an IPW estimator (M5 - M6). The true value of the treatment effect was zero.

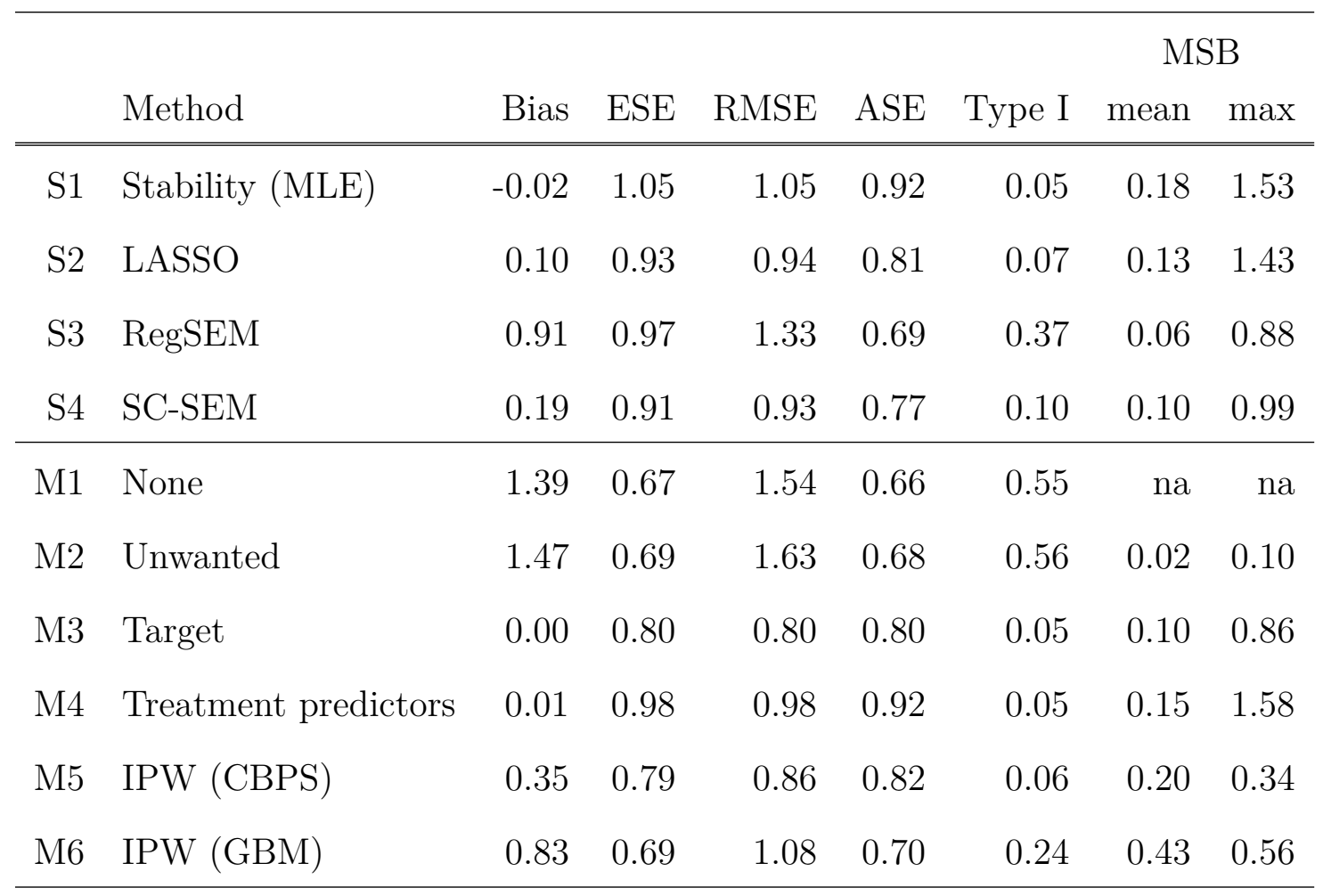

Note. The empirical SE ("ESE") of the effect point estimates (i.e., standard deviation around the average over all simulated datasets), and asymptotic SE ("ASE"; average of the standard error estimates over all simulated datasets), were considered. The root mean squared error ("RMSE") of the effect estimates was calculated as the square root of the mean of squares of the estimates centered at the true value (of zero). The (empirical) type I error rate was the proportion of p-values below 0.05. The mean and maximum across all simulated datasets of the maximum standardized bias ("MSB") among all covariates (in each simulated dataset) were calculated for methods using inverse probability weights; this was non-applicable to the M1 method and simply denoted by "na." All results were rounded to two decimal places. 
selection methods cannot distinguish between confounders - associated with both treatment and outcome - and pure predictors of outcome (or treatment) based on a single regression model. As expected, the frequent omission of confounders from the selected covariates for adjustment led to biased estimates and inflated type I error rates empirically. Lastly, the IPW estimators using either CBPS (M5) or GBM (M6) for the propensity scores, conditioning on all available covariates, were biased under all considered scenarios, notwithstanding strong ignorability being satisfied given the covariates. 14

\section{Summary of simulation studies}

As demonstrated empirically above, the introduced stability method correctly selected (exactly both) confounders for confounding adjustment more frequently than routine variable selection methods which were applied to the outcome model. Inference for the treatment effect given the selected covariates yielded empirically unbiased estimates, and type I error rates which may be (slightly) elevated above their nominal levels empirically. The latter occurs because the estimated variance does not reflect the uncertainty brought about by carrying out data-driven covariate selection using the same data (Huang, 2020b). When seeking approximately valid hypothesis tests of the null of no (individual) treatment effect, the proposed stability method can be enhanced by additionally using full matching (Hansen, 2004, Stuart, 2010) on a propensity score model containing only the selected covariates, then conducting randomization inference using the constructed strata (Rosenbaum, 2002); such a combination has been shown to control the type I error rate empirically (Loh \& Vansteelandt, 2021a).

\footnotetext{
14 The magnitude of the empirical biases using CBPS tended to be smaller than those using GBM. This would be expected because the data-generating process assumed a log-linear model for the treatment with main effects for the covariates, which was correctly specified for the CBPS method, but inappropriate for the GBM method designed to detect non-linear relationships (Setodji et al., 2017); also see, e.g., Cooper and Richardson (1986). Comparisons of IPW estimators using either CBPS or GBM under various non-linear conditions were made by Setodji et al. (2017), and are beyond the scope of this article.
} 


\section{Illustration with applied examples and a practical guide}

The goal of this section is twofold. First, we illustrate the introduced confounder selection strategy using two publicly available real-world datasets. For each dataset, the stability method (S1 in the simulation study) was applied to select covariates for adjustment. For comparison, each of the routine variable selection methods (S2-S4 in the simulation study) was also applied to select covariates. The estimated average treatment effect given only the selected covariates following each of the above methods was then calculated. For completeness, the estimates adjusting for either none or all of the available covariates were also calculated. Second, to help researchers implement the method in practice, we have put together a set of user-friendly $R$ functions which are freely available online 15 Here, we provide a detailed step-by-step practical guide demonstrating how to use the $\mathrm{R}$ functions.

\section{Social interaction quantity on psychological well-being}

Before applying the proposed method to a non-experimental study, we will first illustrate the method using data from a randomized experiment. In a randomized experiment, confounding of the treatment-outcome relation is unlikely and strong ignorability can be reasonably assumed to hold without conditioning on any covariates. Therefore, the treatment effect estimator should not systematically change after adjusting for different covariate subsets (because strong ignorability is satisfied by design) and the resulting trajectory should appear stable. This is exactly what we found, as we demonstrate below.

The dataset was from a study investigating the effect of social interaction quantity on well-being (Ren et al., 2022), and is publicly available through the Open Science Framework (https://osf.io/8r7gz/). A sample of $n=157$ college students from a research university in the United States participated in this study. Participants were randomly assigned to receive a description of a job in which social interactions were either "rarely

15 https://github.com/wwloh/covariate-selection-effect-stability 
involved" (low quantity), "involved to some extent" (moderate quantity), or "constantly involved" (high quantity). For simplicity, we combined the moderate and high quantity groups into a single treated group $(A=1)$ and used the low quantity group as an untreated group $(A=0)$. After randomization, participants reported their anticipated life satisfaction if having the job described to them, using five items (e.g., "In most ways my life would be close to my ideal." on a 7 -point scale $(1=$ strongly disagree; $7=$ strongly agree). We defined the continuous outcome of interest $Y$ to be the average of the five items measuring life satisfaction rescaled to be between zero and one (by subtracting one and dividing by seven). We considered the following pretreatment covariates collected as part of the survey (in total seven covariates): demographic information (age, gender), and the Big Five personality scores (Extraversion, Agreeableness, Conscientiousness, Negative emotionality, and Openess).

The first few rows of the dataset are displayed below:

\begin{tabular}{|c|c|c|c|c|c|c|c|c|c|}
\hline & i Age & Female & Extra & Agree & Consci & NegEmo & Open & treat & \\
\hline \# 1 & 17 & 0 & -0.89 & -0.33 & -1.41 & -0.01 & 0.65 & 1 & 0.51 \\
\hline 2 & 18 & 0 & -1.76 & -0.44 & $-1 \cdot 30$ & 0.86 & -0.55 & 1 & 0.71 \\
\hline 3 & 18 & 0 & -1.51 & -0.89 & -0.52 & -0.89 & 0.15 & 0 & 0.11 \\
\hline & 18 & 0 & -1.01 & -0.89 & -0.63 & 1.49 & 0.55 & 1 & \\
\hline
\end{tabular}

Here and throughout, a logistic regression model was assumed for the binary treatment, and a linear regression model was assumed for the continuous outcome; both models included only the main effects for the predictors solely to simplify illustrating the proposed covariate selection strategy. First, we ordered the covariates using the function ForwardSelect_DS, where the three arguments are the vector of outcomes Y; the matrix of covariates $\mathrm{X}$; and the vector of treatments $\mathrm{A}$, respectively.

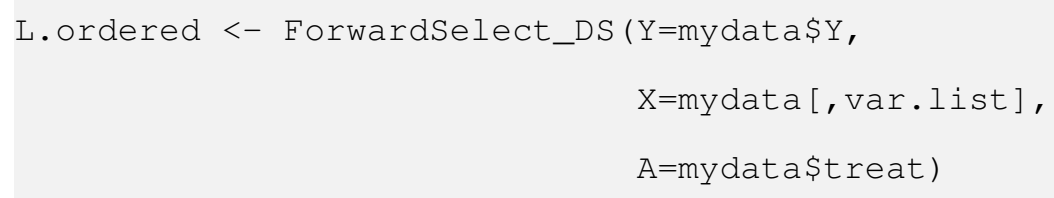

The ordered covariates are printed below and also displayed in Table 2. 
\# [1] "NegEmo" "Extra" "Age" "Consci" "Female" "Agree" "Open"

Next, with the ordered covariates in hand, we calculated the standardized differences between the DR-AIPW estimators (1) for each subset (versus the largest subset with all covariates), using the function StdDiffEst_Ordered_DRAIPW. The first argument is the vector of names for the ordered covariates, and the second argument is the name of the dataset.

L.stable <- StdDiffEst_Ordered_DRAIPW(L.ordered\$ordered, mydata)

We display the (unstandardized) differences in the estimates, i.e., the numerator of (1), and their standard errors, i.e., the denominator of (1), as follows.

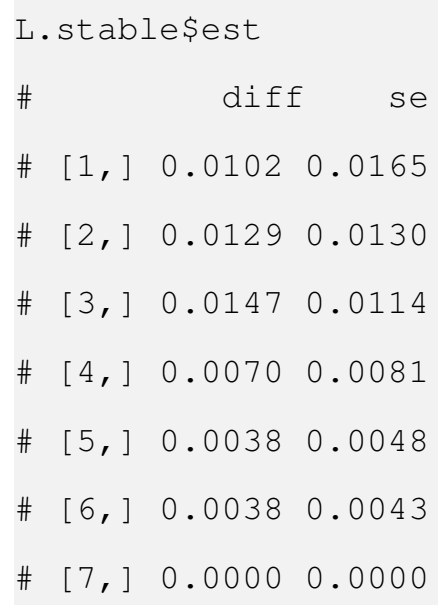

Note that the last row is zero by construction because the differences are with respect to the largest subset with all available covariates. The values of the standardized differences in the estimates are plotted in Figure 2. The function also returns the number of covariates selected using the numerical diagnostic in Appendix A.

L.stable\$selected_orbit

\# [1] 6

The selected subset containing six covariates is indicated by the filled circle in Figure 2 .

Lastly, we can readily calculate the DR-AIPW estimator given the selected covariates using the OneDR_AIPW_Est function. The first argument is the vector of names 
for the selected covariates, the second argument is the name of the dataset, and the third argument is whether to return standard errors.

est <- OneDR_AIPW_Est ( Ls=L.names. selected, mydata, return.se=TRUE)

round (est $[1: 3], 2)$

\# $\quad$ EST $\quad$ SE $\quad$ PV

$\begin{array}{llll}\# \quad 0.23 & 0.05 \quad 0.00\end{array}$

We also display the selected covariates below, noting that they are the first six ordered covariates.

\# [1] "NegEmo" "Extra" "Age" "Consci" "Female" "Agree"

The effect estimates, as well as their accompanying 95\% CIs, adjusting for the covariates in each nested subset are plotted in Figure 3.

For comparison, we applied LASSO (S2), RegSEM (S3), and SC-SEM (S4) to the outcome model; the selected covariates using these methods are shown in Table 2 . The outcome model could not be fitted successfully using SC-SEM (S4). The DR-AIPW estimator given the covariates selected using each method, as well as adjusting for either no covariates or all available covariates were calculated, and results presented in the bottom rows of Table 2. As expected for a randomized experiment, the results were very similar across all the methods, with the $95 \%$ CIs for the treatment effect having similar widths and all excluding zero. 


\section{Figure 2}

Standardized difference ("Std. Diff.;" (1)), between the treatment effect estimator from each subset and from the largest subset, for the Social Interaction data.

\section{Life Satisfaction}

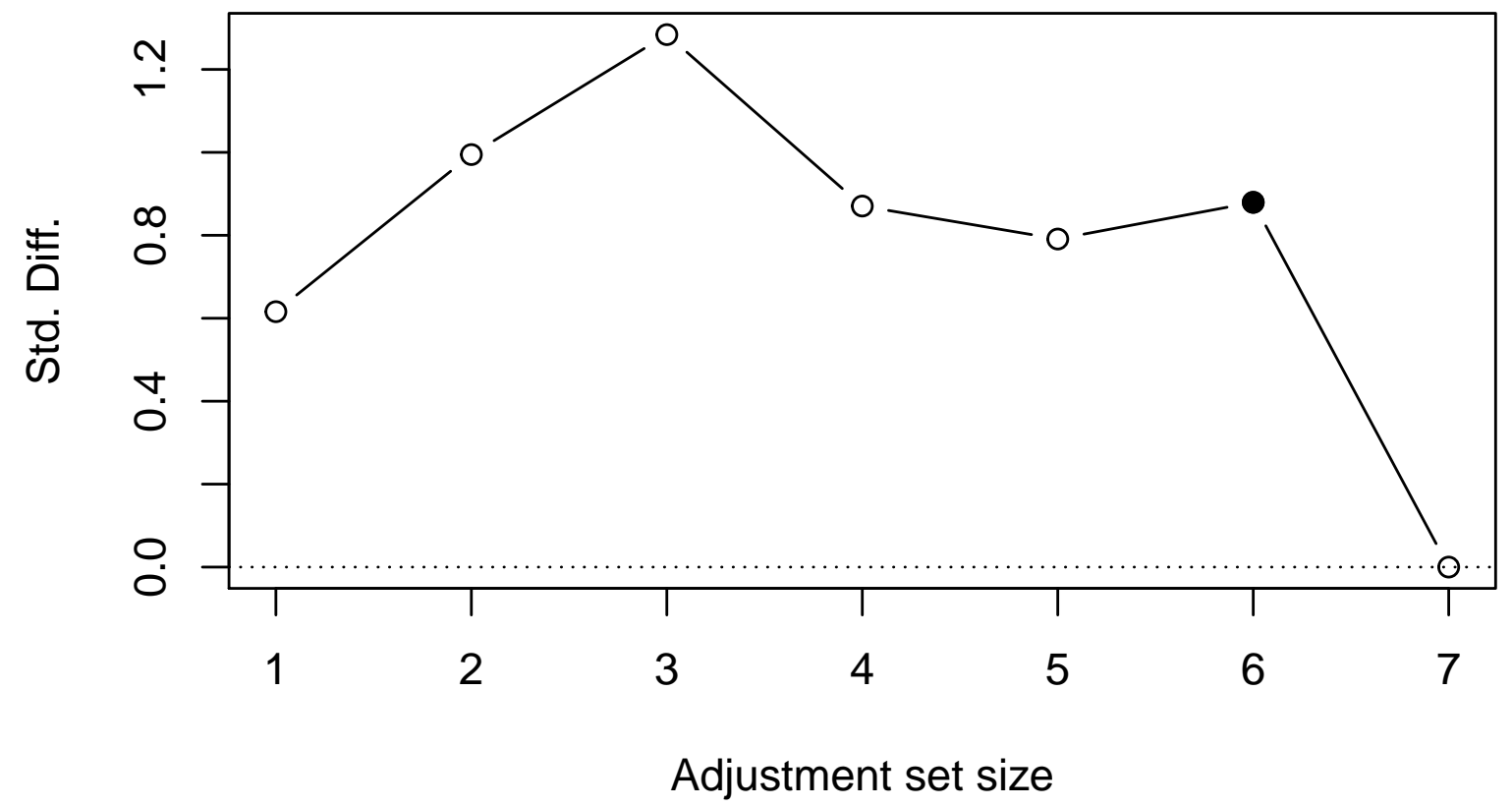

Note. The covariates were ordered using the proposed stability-based procedure. A filled circle indicates the subset with the most stable effect estimator. 


\section{Figure 3}

The DR-AIPW estimate adjusting for the nested covariate subsets indexed by the ordered covariates, for the Social Interaction data.

\section{Life Satisfaction}

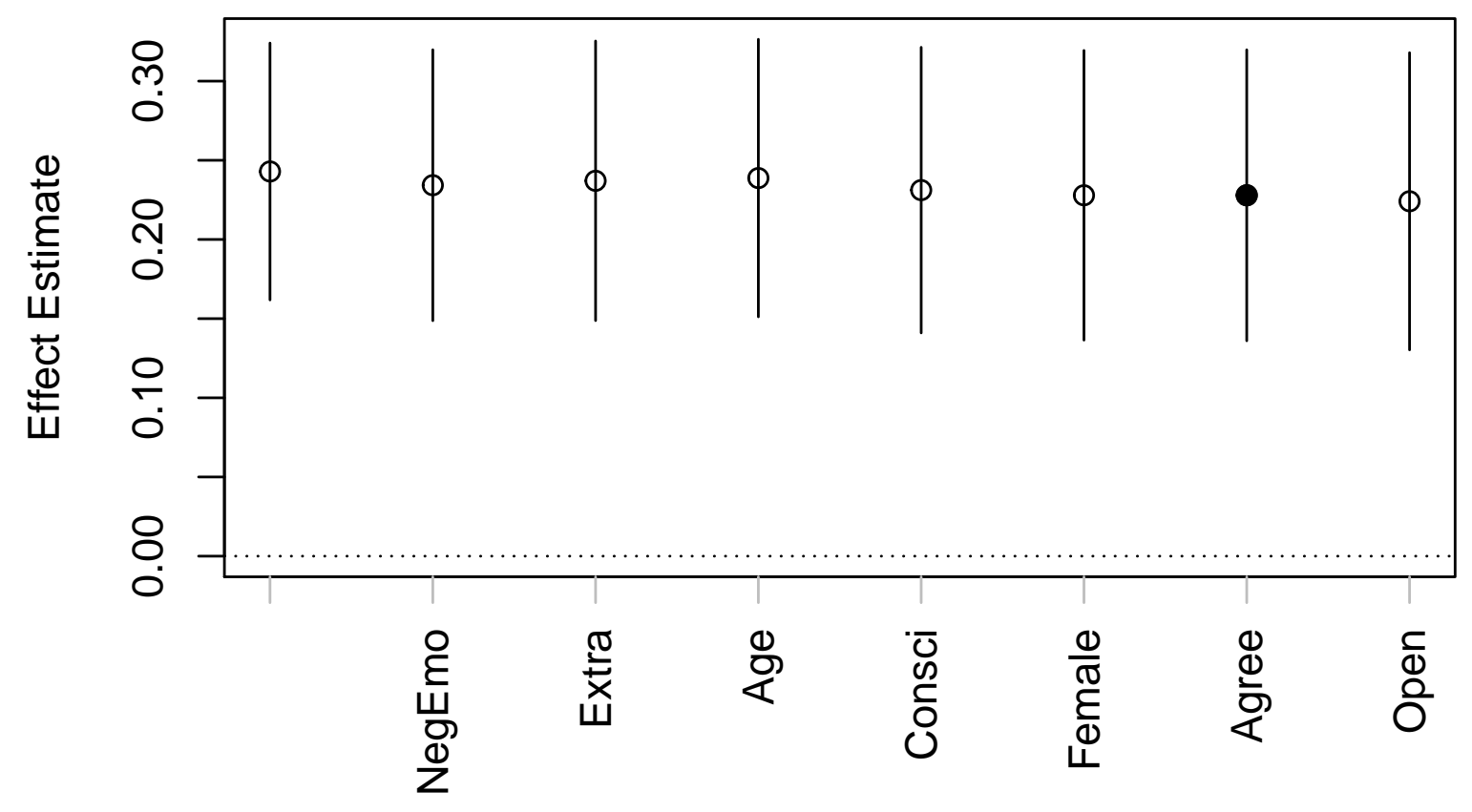

Note. The covariates were ordered using the proposed stability-based procedure. A filled circle indicates the subset with the most stable effect estimator. The vertical lines indicate $95 \%$ CIs. 


\section{Table 2}

Comparison of the selected confounders $(\checkmark)$ in the Social Interaction data using different methods. The covariates were ordered in decreasing priority based on the first step of the proposed stability-based procedure.

\begin{tabular}{|c|c|c|c|c|c|c|}
\hline Covariates & Stability & LASSO & RegSEM & SC-SEM & Empty & All \\
\hline \hline Negative emotionality & $\checkmark$ & & & & & $\checkmark$ \\
Extraversion & $\checkmark$ & & $\checkmark$ & & & $\checkmark$ \\
Age & $\checkmark$ & & $\checkmark$ & & & $\checkmark$ \\
Conscientiousness & $\checkmark$ & & & & & $\checkmark$ \\
Gender & $\checkmark$ & & & & & $\checkmark$ \\
Agreeableness & $\checkmark$ & & & & & $\checkmark$ \\
Openness & & & & & & \\
Treatment effect estimate & 0.23 & 0.24 & 0.25 & 0.24 & 0.24 & 0.22 \\
Standard error & 0.05 & 0.04 & 0.04 & 0.04 & 0.04 & 0.05 \\
95\% CI (upper endpoint) & 0.32 & 0.32 & 0.33 & 0.32 & 0.32 & 0.32 \\
95\% CI (lower endpoint) & 0.14 & 0.16 & 0.17 & 0.16 & 0.16 & 0.13 \\
\hline
\end{tabular}

Note. The estimated average (marginal) treatment effect, standard error, and $95 \%$ CI are stated in the bottom four rows. The results adjusting for either no covariates ("Empty"), or all the available covariates ("All") are shown in the two rightmost columns. All results were rounded to two decimal places. 


\section{New math curriculum on test scores}

We now illustrate the proposed method using data from an observational study with a non-randomly assigned treatment so that confounding of the treatment-outcome relationship was plausible. The dataset was from a study conducted to evaluate the impact of a new math curriculum on students' math performance (Powell et al., 2020), and is publicly available through the Open Science Framework (https://osf.io/8t3s9/). Students in grades 9 through 12 across three schools were instructed in a particular academic year using either a new math curriculum (treatment $A=1 ; 166$ students), or the traditional curriculum (control $A=0 ; 159$ students). The new math curriculum was non-randomly assigned, and the choice of which curriculum to adopt at the start of the academic year depended on the teacher: three teachers (in two of the three schools) adopted the new math curriculum and four teachers (across the three schools) used the traditional curriculum. For simplicity of presenting the procedure proposed in this paper, we will follow Powell et al. (2020) and ignore the clustered data structure; extensions to incorporate teacher- and school-level effects (Fuentes et al., 2021; Thoemmes \& West, 2011) are deferred to future work. The continuous outcome of interest $Y$ was students' scores from a standardized elementary algebra test that was administered at the end of the academic year. We considered the following pretreatment covariates collected at the start of the academic year (in total 18 covariates): demographic information (race, grade, gender, expected math grade for the year), and scores from separate scales of three standardized tests (elementary algebra, mathematical problem solving profiles, and perceived attitude towards mathematics). For the sole purpose of illustrating the proposed method, we merely assumed that strong ignorability was met given these measured covariates. In practice, researchers should use established theory and expert knowledge to critically assess whether the strong ignorability assumption can reasonably hold for their study.

The first few rows of the dataset are displayed below:

\# $\quad$ i Sex Grade GradeExp AfAm AmInd Cauc MexAm AsPIFi PR Other AikPos 


\begin{tabular}{|c|c|c|c|c|c|c|c|c|c|c|c|c|c|c|}
\hline \# & 1 & 1 & 9 & & 4 & 0 & 0 & 1 & & 0 & 0 & 0 & & 0 \\
\hline \# & 2 & 2 & 9 & & 4 & 0 & 0 & 1 & & 0 & 0 & 0 & & 0 \\
\hline \# & 3 & 3 & 9 & & 4 & 0 & 0 & 1 & & 0 & 0 & 0 & & 0 \\
\hline \# & 4 & 4 & 9 & & 3 & 0 & 0 & 1 & & 0 & 0 & 0 & & 0 \\
\hline \# & & $\mathrm{AikNe}$ & 9 CRNum & CRAlg & $\mathrm{CR}$ & pace & CRMeas & CRCD & & retest & trea & & Y & \\
\hline \# & 1 & 1 & 1 & 2 & & 2 & 2 & 1 & & 23 & & 0 & 32 & \\
\hline \# & 2 & 1 & 4 & 1 & & 3 & 2 & 1 & & 23 & & 0 & 32 & \\
\hline \# & 3 & 2 & 2 & 1 & & 2 & 2 & 1 & & 24 & & 0 & 38 & \\
\hline \# & 4 & 3 & 1 & 1 & & 2 & 2 & 1 & & 24 & & 0 & 33 & \\
\hline
\end{tabular}

As demonstrated in the first data example, the analysis proceeds in three simple steps. First, we ordered the covariates using the function ForwardSelect_DS.

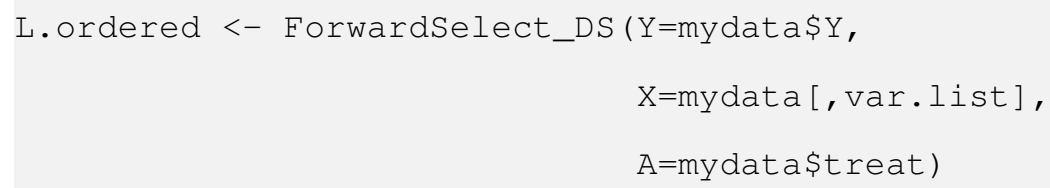

The ordered covariates are printed below and also displayed in Table 3

$\begin{array}{llcccc}\# \text { [1] "Pretest" } & \text { "GradeExp" "AfAm" } & \text { "Cauc" } & \text { "AikPos" } & \text { "Grade" } \\ \text { \# [7] "CRAlg" } & \text { "AikNeg" } & \text { "CRMeas" } & \text { "CRSpace" } & \text { "Other" } & \text { "AmInd" } \\ \text { \# [13] "PR" } & \text { "MexAm" } & \text { "CRCD" } & \text { "AsPlfi" } & \text { "CRNum" } & \text { "Sex" }\end{array}$

Next, we calculated the standardized differences between the DR-AIPW estimators (1) for each subset (versus the largest subset with all covariates), using the function StdDiffEst_Ordered_DRAIPW.

L.stable <- StdDiffEst_Ordered_DRAIPW(L.ordered\$ordered, mydata)

We display the differences in the estimates and their standard errors as follows.

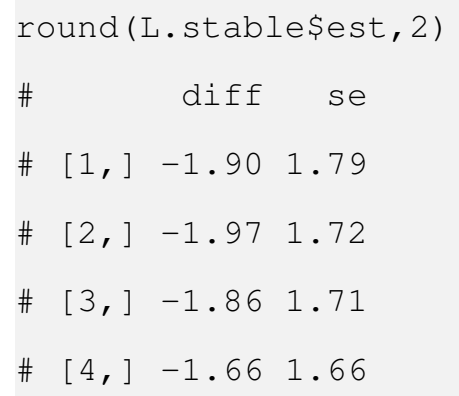


\# $[5]-,1.64 \quad 1.63$

\# $[6]-,1.83 \quad 1.50$

\# [7, ] $-2.44 \quad 1.59$

$\begin{array}{llll}\#[8,] & -2.06 & 1.37\end{array}$

\# $[9] \quad-,1.47 \quad 1.21$

$\#[10]-,1.401 .20$

\# $[11]-,0.420 .27$

$\#[12]-,0.110 .27$

\# $[13]-,0.26 \quad 0.22$

\# $[14]-,0.310 .21$

\# $[15]-,0.22 \quad 0.17$

\# $[16]-,0.120 .12$

\# $[17] \quad$,

$\#[18] \quad 0.00 \quad$,

The values of the standardized differences in the estimates are plotted in Figure 4. The function also returns the number of covariates selected.

L.stable\$selected_orbit

\# [1] 2

The selected covariate subset is indicated by the filled circle in Figure 4 .

Lastly, we calculate the DR-AIPW estimator given the selected covariates using the OneDR_AIPW_Est function.

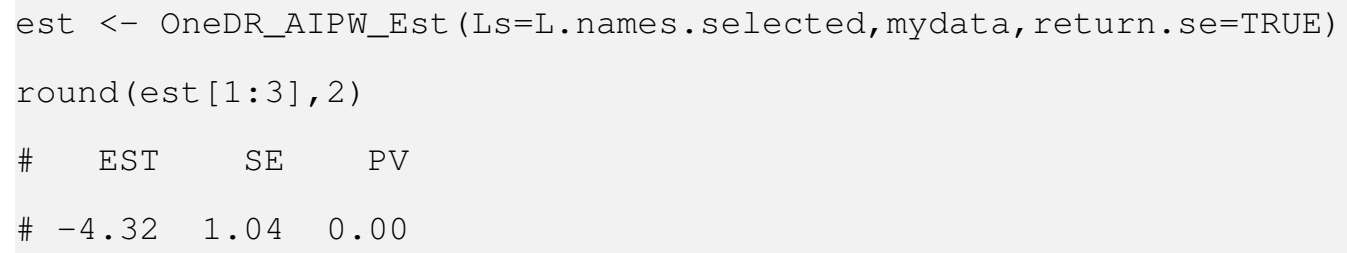

We also display the selected covariates below, noting that they are the first two ordered covariates.

\# [1] "Pretest" "GradeExp" 
The effect estimates, as well as their accompanying 95\% CIs, adjusting for the covariates in each nested subset are plotted in Figure 5.

As in the previous example, for comparison, we applied each routine variable selection method (S2, S3, and S4) to the outcome model, but SC-SEM (S4) failed to converge. The selected covariates using each method under comparison are also shown in Table 3. The DR-AIPW effect estimator given the covariates selected using each method, as well as adjusting for either no covariates or all available covariates were calculated, and results presented in the bottom rows of Table 3 . The $95 \%$ CIs for the treatment effect all excluded zero following data-driven covariate or variable selection, whereas the $95 \% \mathrm{CI}$ adjusting for all available covariates was much wider and included zero. Visual inspection of the effect estimates in Figure 5 revealed that the point estimates maintained a relatively stable negative value even after adjusting for all the covariates.

Visualizing the trajectory of the effect estimates is, therefore, an advantageous feature of the method. Such a visual inspection offers direct insight into the (in)sensitivity of the estimator adjusting for various covariate subsets. As shown in this applied example, results following a data-driven covariate selection procedure may differ from results following routine adjustment for all available putative confounders. Under such scenarios, we recommend researchers reexamine their fitted estimation models and theoretical justifications for including various variables for confounding adjustment. 


\section{Figure 4}

Standardized difference ("Std. Diff.;" (1)), between the treatment effect estimator from each subset and from the largest subset, for the Math data.

\section{New Math Curriculum}

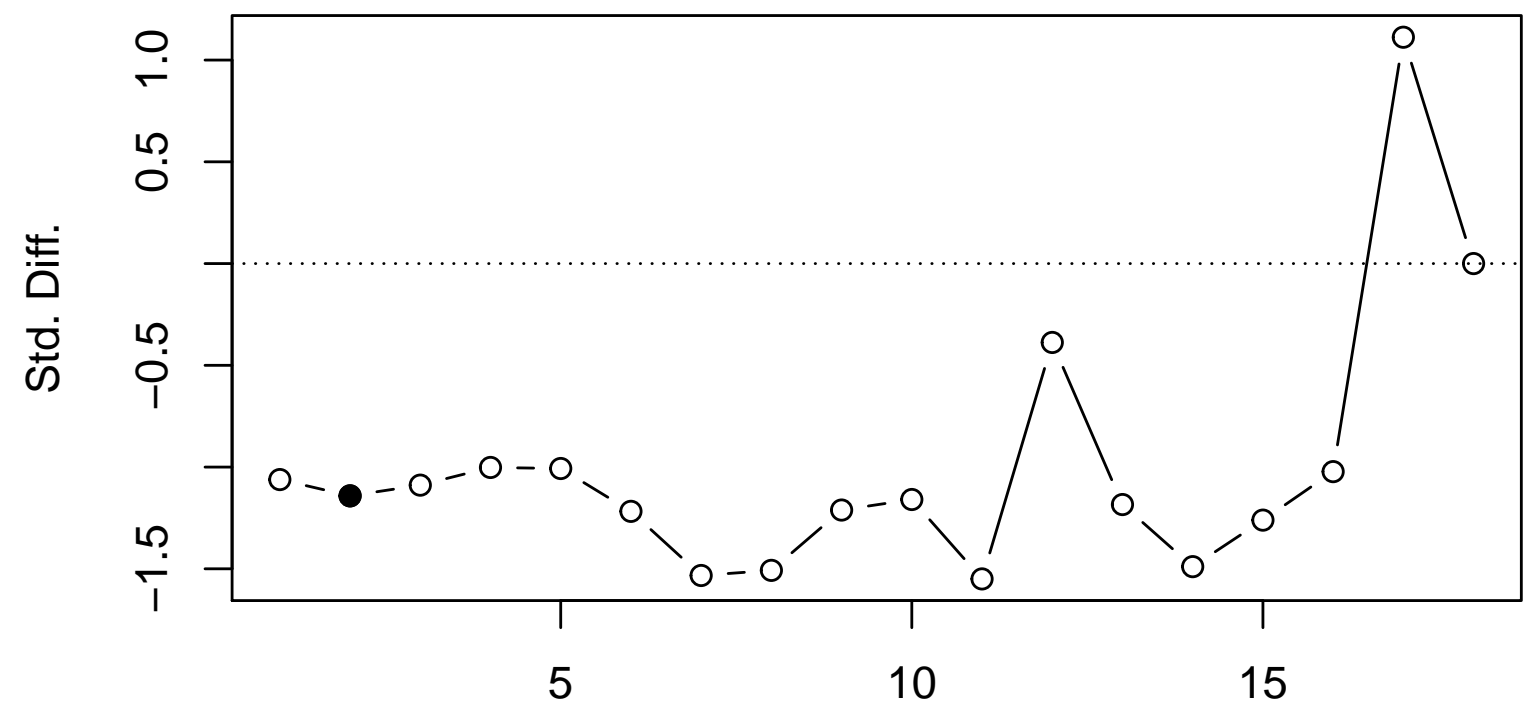

Adjustment set size

Note. The covariates were ordered using the proposed stability-based procedure. A filled circle indicates the subset with the most stable effect estimator. 


\section{Figure 5}

The DR-AIPW estimate adjusting for the nested covariate subsets indexed by the ordered covariates, for the Math data. The vertical lines indicate $95 \%$ CIs.

\section{New Math Curriculum}

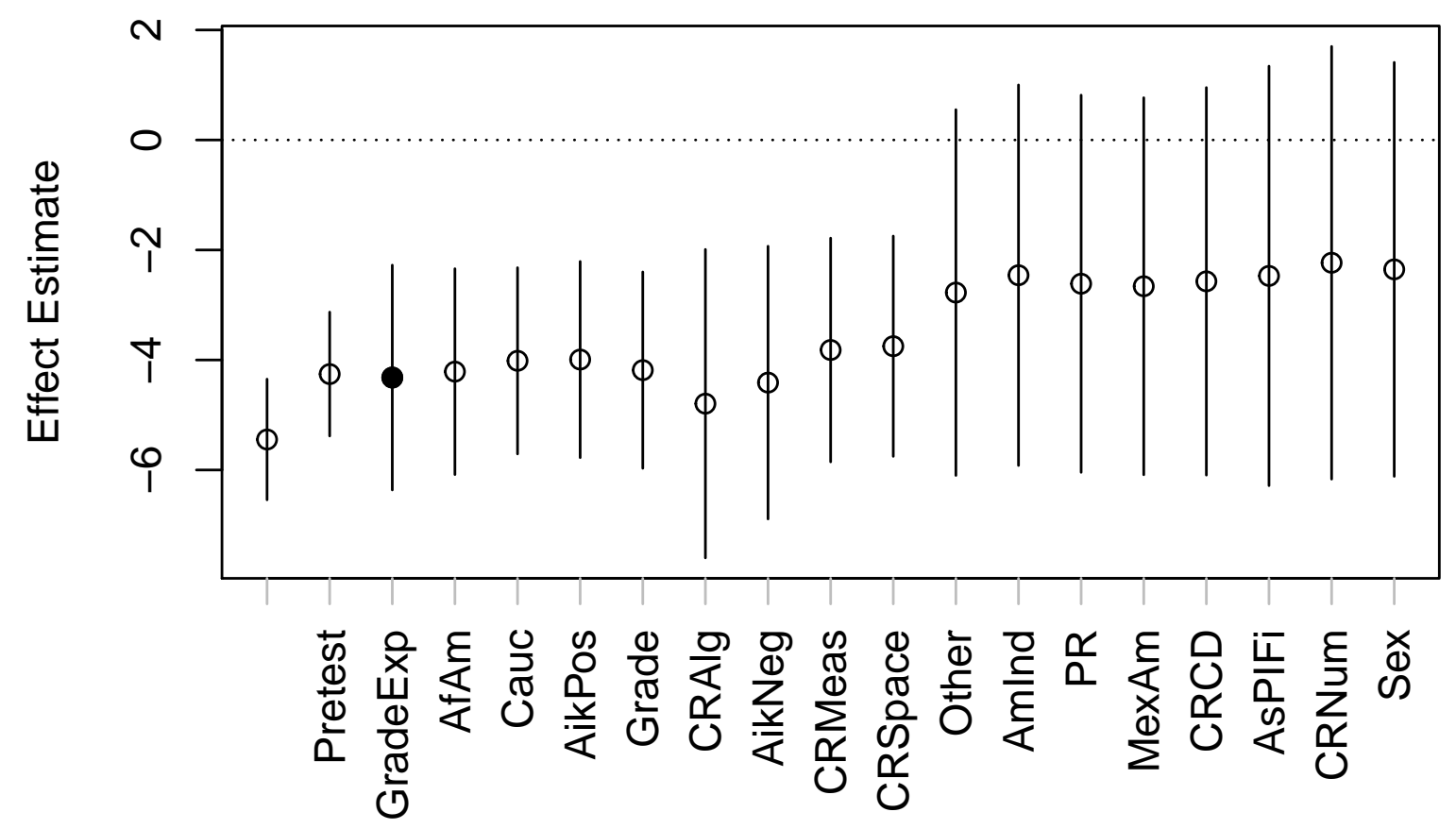

Note. The covariates were ordered using the proposed stability-based procedure. A filled circle indicates the subset with the most stable effect estimator. The vertical lines indicate $95 \%$ CIs. 


\section{Table 3}

Comparison of the selected confounders $(\checkmark)$ in the Math data using different methods. The covariates were ordered in decreasing priority based on the first step of the proposed stability-based procedure.

\begin{tabular}{|c|c|c|c|c|c|c|}
\hline Covariates & Stability & LASSO & RegSEM & SC-SEM & Empty & All \\
\hline Elementary algebra test score at baseline (Pretest) & $\checkmark$ & $\checkmark$ & $\checkmark$ & & & $\checkmark$ \\
\hline Expected mathematics grade (GradeExp) & $\checkmark$ & $\checkmark$ & & & & $\checkmark$ \\
\hline African American (AfAm) & & $\checkmark$ & & & & $\checkmark$ \\
\hline Caucasian (Cauc) & & $\checkmark$ & & & & $\checkmark$ \\
\hline Mathematics attitude scale: Positive (AikPos) & & & $\checkmark$ & & & $\checkmark$ \\
\hline Grade in school (Grade) & & $\checkmark$ & & & & $\checkmark$ \\
\hline Mathematical profile scores: Algebra (CRAlg) & & $\checkmark$ & & & & $\checkmark$ \\
\hline Mathematics attitude scale: Negative (AikNeg) & & $\checkmark$ & $\checkmark$ & & & $\checkmark$ \\
\hline Mathematical profile scores: Measurement (CRMeas) & & $\checkmark$ & & & & $\checkmark$ \\
\hline Mathematical profile scores: Space (CRSpace) & & $\checkmark$ & & & & $\checkmark$ \\
\hline None of the other race categories (Other) & & & & & & $\checkmark$ \\
\hline American Indian (AmInd) & & & & & & $\checkmark$ \\
\hline Puerto Rican (PR) & & & & & & $\checkmark$ \\
\hline Mexican American (MexAm) & & $\checkmark$ & & & & $\checkmark$ \\
\hline Mathematical profile scores: Chance and Data (CRCD) & & & & & & $\checkmark$ \\
\hline Asian/Pacific Islander (AsPIFi) & & & & & & $\checkmark$ \\
\hline Mathematics Profile Scores: Number (CRNum) & & $\checkmark$ & & & & $\checkmark$ \\
\hline Gender (Sex) & & & & & & $\checkmark$ \\
\hline Treatment effect estimate & -4.32 & -3.86 & -4.06 & -5.45 & -5.45 & -2.35 \\
\hline Standard error & 1.04 & 0.95 & 0.61 & 0.56 & 0.56 & 1.92 \\
\hline 95\% CI (upper endpoint) & -2.28 & -2.01 & -2.87 & -4.35 & -4.35 & 1.41 \\
\hline 95\% CI (lower endpoint) & -6.36 & -5.71 & -5.26 & -6.54 & -6.54 & -6.12 \\
\hline Median standardized bias & 0.43 & 0.09 & 0.03 & na & na & 0.18 \\
\hline Maximum standardized bias & 0.66 & 0.77 & 0.05 & na & na & 0.71 \\
\hline
\end{tabular}

Note. The estimated average (marginal) treatment effect, standard error, 95\% CIs, and median and maximum standardized bias among all (selected) covariates are stated in the bottom six rows. Methods that did not adjust for any covariates had standardized biases as "na." The results adjusting for either no covariates ("Empty"), or all the available covariates ("All") are shown in the two rightmost columns. All results were rounded to two decimal places. 


\section{Discussion}

In this article, we introduced a data-driven confounder selection strategy that exploits evaluating the impact of covariate selection on the quality of the effect estimator. In particular, we utilize the causal knowledge that after adjusting for covariates that suffice to eliminate all confounding, the addition of non-confounders associated with only either treatment or outcome, but not both, should not systematically alter the effect estimator.

\section{Step 1: Prioritizing the covariates for confounding adjustment}

In the first step of the strategy, covariates are prioritized for confounding adjustment. For ease of exposition, in this paper, we evaluated their (partial) associations in regression models with main effects only, then used the corresponding p-values to measure their relative importance for prioritizing them. However, it is conceptually straightforward to generalize this step by allowing for any other pre-defined importance criterion based on a researcher's determination. We offer a few suggestions of different possibilities that are exciting avenues for future research.

First, we can relax the assumption of only main effects when assessing the conditional associations, e.g., in steps A1 and A3. Non-linear higher-order or interaction terms among the predictors can be readily fitted. For example, an interaction term between $L_{2}$ and either $L_{1}$ or $L_{3}$ can be included in the logistic regression model for the treatment in step A3(a) and the linear regression model for the outcome in step A3(b), respectively:

$$
\begin{aligned}
\operatorname{logit}\left\{\mathrm{E}\left(A \mid \mathcal{L}^{1}, L_{k}\right)\right\} & =\alpha_{1,0}+\alpha_{1,2} L_{2}+\alpha_{1, k} L_{k}+\alpha_{1,2 \times k} L_{2} L_{k} ; k=1,3 ; \\
\mathrm{E}\left(Y \mid A, \mathcal{L}^{1}, L_{k}\right) & =\beta_{1,0}+\beta_{1, A} A+\beta_{1,2} L_{2}+\beta_{1, k} L_{k}+\beta_{1,2 \times k} L_{2} L_{k} ; k=1,3 .
\end{aligned}
$$

A likelihood ratio test of the null hypothesis that $\alpha_{1, k}=\alpha_{1,2 \times k}=0$ can then be used to calculate a p-value for each candidate covariate in the treatment model. Similarly, a likelihood ratio test of the null hypothesis that $\beta_{1, k}=\beta_{1,2 \times k}=0$ can be used to calculate a p-value for each candidate covariate in the outcome model. Denote the resulting p-values by $p v\left(A ; \mathcal{L}^{1}, L_{k}\right)$ and $p v\left(Y ; \mathcal{L}^{1}, L_{k}\right)$ respectively. Then in step $\mathrm{A} 4$, add the covariate with 
the smallest p-value among the four p-values $\left(p v\left(A ; \mathcal{L}^{1}, L_{k}\right), p v\left(Y ; \mathcal{L}^{1}, L_{k}\right)\right), k=1,3$, across both treatment and outcome models, to the subset $\mathcal{L}^{2}$. However, such non-linear terms can be incorporated only if researchers specify them in advance. Machine learning algorithms, such as random forests (Athey \& Wager, 2019, Breiman, 2001) and boosting (McCaffrey et al., 2004), can automatically incorporate non-linear functions of the covariates in the outcome or treatment models. The covariates could then be prioritized using nonparametric variable importance measures (Fisher et al., 2019; Williamson et al., 2021).

Second, we adopted p-values (e.g., in steps A2 and A4) because they are simple measures of standardized conditional associations that facilitate comparisons with other candidate covariates on a common scale. In practice, observations often have partially recorded data, so p-values based on a complete case analysis can be sensitive to the sample sizes. Hence, future work can explore incorporating missing data methods, such as full information maximum likelihood or multiple imputation (Dong \& Peng, 2013; Lee \& Shi, 2021). Alternatively, other importance metrics that avoid using p-values can be considered. For example, one can exploit the relative contribution of each covariate towards eliminating the observed confounding bias following Parast and Griffin (2020). Another example is to use dominance analysis to rank the relative predictive ability of predictors in a multiple linear regression (Azen \& Budescu, 2003) for the outcome model when the outcome is continuous. Another possibility, following the traditional "design of observational studies" in the propensity score literature (Rubin, 2007; Stuart \& Rubin, 2008), is to exploit relative measures of covariate balance without using empirical information about the outcome. Similarly, separate analyses of different parts of the treatment and outcome models, thus concealing information in one part from the other parts (Cook et al., 2009; Cook et al., 2020; Steiner et al., 2010 Zurovaca et al., 2021), could then be combined to obtain relative measures of importance for each covariate ${ }^{16}$

Third, in the latter applied example, following Powell et al. (2020), the "race"

${ }^{16}$ We thank a reviewer for offering these suggestions. 
covariate was coded as a series of auxiliary binary (dummy) variables, in line with how non-ordinal categorical variables are typically included as predictors in regression models. This form of coding can potentially lead to selecting only certain levels of the original categorical variable, which changes the interpretation of the selected variables (see Fox and Monette, 1992 for a related issue in the context of multicollinearity). If interest is in maintaining the interpretability of the original categorical variable during covariate prioritization, then the procedure can be readily modified by considering all the associated auxiliary variables as a group; see, e.g., Tutz and Gertheiss (2016) and Yuan and Lin (2006) for related methods in the context of variable (or "feature") selection.

\section{Step 2: Evaluating the stability of the effect estimator}

In the second step of the strategy, given a hierarchical ordering of the covariates, the effect estimator adjusting for each implied subset is calculated. We proposed using a DR-AIPW estimator endowed with various desirable properties (Glynn \& Quinn, 2010: Kang \& Schafer, 2007; Kurz, 2022, Zhong et al., 2021). In principle, researchers can readily utilize any effect estimator (e.g., using only either a treatment model or an outcome model) to assess stability and subsequently draw post-selection inferences about the treatment effect. Because the proposal requires adjusting for a series of nested covariate subsets, we prefer effect estimators with closed-form expressions for the variance estimator, over estimators that demand computationally intensive nonparametric resampling procedures, to ease calculating the variance estimator in the denominator of (1).

Even when it can be reasonably assumed that strong ignorability holds given a set of available putative confounders, in most realistic settings, it is impossible to determine whether any single subset remains sufficient to eliminate confounding. In practice, researchers may already be informally considering different covariate subsets as a form of post-hoc sensitivity analysis simply by re-estimating the causal effect using different covariates based on updated knowledge or inputs from editors or reviewers. This can 
potentially introduce subjectivity into the analysis (Ioannidis, 2005, 2007) ${ }^{17}$ Therefore, an advantage of the proposal is explicating the steps a researcher takes first to prioritize, and subsequently include covariates for confounding adjustment in a transparent manner. The combined use of double selection to prioritize the covariates, and stability-based evaluation of the effect estimator to select a subset, is intended to strengthen the justification that adjusting for the chosen covariates suffices to eliminate all observed confounding biases, assuming strong ignorability holds given the available covariates.

\section{Bias-variance tradeoff}

The motivation for using the double selection criterion in the first step is to minimize confounding bias. By exploiting empirical information on the covariate-treatment and covariate-outcome associations simultaneously, we increase the chances of selecting true confounders (i.e., associated with both treatment and outcome). However, such a criterion has the downside of reducing the chances of selecting variables that predict the outcome only. Adjusting for covariates only (or strongly) related to the outcome but un- (or weakly) related to the treatment can improve the finite sample variance of the treatment effect estimator, notwithstanding their inability to reduce confounding bias (Austin et al., 2007; Brookhart et al., 2006) ${ }^{18}$ In contrast, adjusting for non-confounders associated with treatment only and unassociated with outcome is known to adversely increase the effect estimator's variability without reducing confounding bias (Austin et al., 2007; Brookhart

\footnotetext{
17 Researchers are encouraged to include the list of pre-treatment covariates for confounding adjustment as part of their pre-registered analysis plan. A comprehensive selection of pertinent covariates can be collated based on current theoretical and external empirical knowledge, or in consultation with subject matter experts; see Steiner et al. (2010) for detailed recommendations.

18 Even in randomized experiments with no confounding by design, adjusting for pre-treatment or baseline covariates (associated with the outcome) can reduce the effect estimator's variability and improve statistical power; see, e.g., Kahan et al. (2014). For example, ANCOVA is recommended to increase statistical power because the error variance will typically be reduced (van Breukelen, 2013), thereby increasing the precision of the treatment's regression coefficient estimator (Tsiatis et al., 2008).
} 
et al., 2006, Kelcey, 2011; VanderWeele, 2019; Westreich et al., 2011). Adjusting for such non-confounders is unnecessary for eliminating confounding bias yet yields inefficient estimators which can be biased in finite samples, simply due to sampling variability. For these reasons, recent developments in data-adaptive covariate selection (Ju et al., 2020; Koch et al., 2018; Shortreed \& Ertefaie, 2017; Wang et al., 2015) aim to include covariates associated with the outcome, while excluding or penalizing covariates associated with treatment only, albeit raising the risks of confounding biases due to omitted confounders.

Therefore, the proposed procedure adopts a similar approach by further utilizing the standardized differences to evaluate the stability of the effect estimators adjusting for different covariate subsets, while accounting for their variability, in the second step. We observed from the first applied example with a randomized treatment that six non-confounders who improved the precision of the effect estimator were selected, notwithstanding that they were unnecessary for confounding adjustment. Ideally, we would be able to clarify the contribution of adjusting for each additional covariate toward reducing either bias or variance of the effect estimator; e.g., by inspecting unstandardized point estimates and their standard errors, similar to the plots displayed in Figures 3 and 5. But in practice, the true value of the treatment effect is rarely known with certainty (or in the absence of sampling error), which makes calculating the bias unfeasible. Thomas Cook and colleagues (Cook et al., 2009, Cook et al., 2020, Steiner et al., 2015, Steiner et al., 2010; Zurovaca et al., 2021) offer suggestions for what variables should be included for confounding adjustment based on comparisons of randomized experiments and observational studies having a treatment in common. Future work may consider using one of Thomas Cook's data sets to compare treatment effect estimates from a randomized experiment and an observational study sharing a treatment.19

\footnotetext{
19 We thank a reviewer for offering this suggestion.
} 


\section{Other relevant issues}

In the simulation studies, we considered only continuous outcomes and continuous covariates to facilitate comparisons with current variable selection methods for SEM using regularization (Jacobucci et al., 2016) or penalized likelihood (Huang et al., 2017). Simulation studies with a binary outcome and non-continuous covariates were conducted in Loh and Vansteelandt (2021a), who implemented various (data-driven) covariate selection methods from the causal inference literature for comparison. In this paper, we assumed that all covariates were reliably measured. To avoid biases arising from poorly measured predictors, researchers should consider measurement error in the covariates when adjusting for a given subset; see, e.g., Sengewald and Mayer (2022) and Sengewald et al. (2019).

Finally, it is essential to reiterate that, same as other existing data-driven covariate selection methods, the proposal introduced in this paper builds on the assumption that a measured subset of the potential confounders suffices to satisfy strong ignorability. However, confounders may nonetheless remain unmeasured, potentially yielding biased inferences. Therefore, it is a judicious practice to conduct a sensitivity analysis to probe the impact of violations of strong ignorability due to unmeasured confounding; see, e.g., Hong et al. (2021) and Rosenbaum (1987a), as well as others described in Liu et al. (2013). In ongoing work, the proposed approach can be extended to gauge the impact of unmeasured confounding on the conclusions drawn, by extrapolating the fitted trajectory of the effect estimator across measured confounders towards additional hypothetical unmeasured confounders (Loh \& Vansteelandt, 2021b).

\section{A note on practical suggestions}

We have two practical suggestions for applied researchers to consider when applying the proposed strategy. First, we encourage researchers to actively incorporate their theoretical and expert knowledge into the proposed data-driven procedure. For example, researchers should carefully consider the criterion for prioritizing covariates in the first step of the proposal. We recommend taking into account both the covariate-treatment and 
covariate-outcome relations for each covariate. After ordering the covariates empirically, researchers should inspect the results and make further refinements to obtain theoretically meaningful subsets based on substantive knowledge. Second, researchers should judge critically the relatively stability of the effect estimator over different nested covariate subsets in the second step of the proposal. We recommend using both visual inspection and numerical diagnosis to select a sufficient covariate subset. For example, the effect estimator (and corresponding CIs) adjusting for different nested subsets indexed by the prioritized covariates can be plotted, as shown in Figures 3 and 5. Visual inspection permits qualitative examinations of unstandardized effect estimates, and their accompanying standard errors; whereas numerical diagnosis facilitates quantitative optimization of stability that accounts for sampling variability.

\section{Conclusion}

In observational studies where treatment is non-randomized, valid statistical inference of a causal effect is contingent on careful confounding adjustment. Even when the

measured covariates suffice for strong ignorability to hold, merely adjusting for all available covariates may be impossible due to the curse of dimensionality, and even if feasible, can adversely affect the quality of the effect estimator. To avoid such difficulties, data-driven covariate (or confounder) selection procedures have been developed to improve causal effect estimation. But these recent developments - which are routinely applied in other fields are absent in the psychology literature. In this article, we introduced a state-of-the-art data-driven confounder selection strategy that focuses on the stability of the effect estimator. This strategy offers a systematic and principled approach to guide the covariate selection process by synthesizing substantive theory-based covariate selection with empirical evaluation. In the first step, prioritizing covariates for confounding adjustment permits researchers to transparently report and refine each covariate's relative importance. In the second step, gauging the trajectory of the estimator over different subsets, while accounting for its sampling variability, offers direct insight into the (in)sensitivity of the 
estimator to the selected covariate subset. In both steps, quantitative assessment and qualitative evaluation can be systematically carried out. Researchers can therefore gain direct insight into the estimator's stability or (in)sensitivity to the covariates being adjusted for. We encourage psychological researchers seeking to draw causal inferences in observational studies to employ the stability strategy in providing a more resilient justification for eliminating confounding biases. 


\section{References}

Athey, S., \& Wager, S. (2019). Estimating treatment effects with causal forests: An application. Observational Studies, 5(2), 37-51. https://doi.org/10.1353/obs.2019.0001

Austin, P. C. (2018). Assessing the performance of the generalized propensity score for estimating the effect of quantitative or continuous exposures on binary outcomes. Statistics in Medicine, $37(11), 1874-1894$. https://doi.org/https://doi.org/10.1002/sim.7615

Austin, P. C., Grootendorst, P., \& Anderson, G. M. (2007). A comparison of the ability of different propensity score models to balance measured variables between treated and untreated subjects: A monte carlo study. Statistics in Medicine, 26(4), 734-753. https://doi.org/https://doi.org/10.1002/sim.2580

Austin, P. C., \& Stuart, E. A. (2015). Moving towards best practice when using inverse probability of treatment weighting (IPTW) using the propensity score to estimate causal treatment effects in observational studies. Statistics in Medicine, $34(28)$, 3661-3679. https://doi.org/https://doi.org/10.1002/sim.6607

Azen, R., \& Budescu, D. V. (2003). The dominance analysis approach for comparing predictors in multiple regression. Psychological Methods, 8(2), 129-148. https://doi.org/10.1037/1082-989X.8.2.129

Bang, H., \& Robins, J. M. (2005). Doubly robust estimation in missing data and causal inference models. Biometrics, 61(4), 962-973. https://doi.org/10.1111/j.1541-0420.2005.00377.x

Belloni, A., Chernozhukov, V., \& Hansen, C. (2014). Inference on treatment effects after selection among high-dimensional controls. The Review of Economic Studies, 81(2), 608-650. https://doi.org/10.1093/restud/rdt044

Breiman, L. (2001). Random forests. Machine Learning, 45(1), 5-32. 
Brookhart, M. A., Schneeweiss, S., Rothman, K. J., Glynn, R. J., Avorn, J., \& Stürmer, T. (2006). Variable selection for propensity score models. American Journal of Epidemiology, 163(12), 1149-1156. https://doi.org/10.1093/aje/kwj149

Cefalu, M., Ridgeway, G., McCaffrey, D., Morral, A., Griffin, B. A., \& Burgette, L. (2021). Twang: Toolkit for weighting and analysis of nonequivalent groups [R package version 2.0]. https://CRAN.R-project.org/package=twang

Cook, T. D., Steiner, P. M., \& Pohl, S. (2009). How bias reduction is affected by covariate choice, unreliability, and mode of data analysis: Results from two types of within-study comparisons [PMID: 26801798]. Multivariate Behavioral Research, 44 (6), 828-847. https://doi.org/10.1080/00273170903333673

Cook, T. D., Zhu, N., Klein, A., Starkey, P., \& Thomas, J. (2020). How much bias results if a quasi-experimental design combines local comparison groups, a pretest outcome measure and other covariates?: A within study comparison of preschool effects. Psychological Methods, 25(6), 726-746. https://doi.org/10.1037/met0000260

Cooper, W. H., \& Richardson, A. J. (1986). Unfair comparisons. Journal of Applied Psychology, 71, 179-184. https://doi.org/10.1037/0021-9010.71.2.179

Dong, Y., \& Peng, C.-Y. J. (2013). Principled missing data methods for researchers. SpringerPlus, 2(1), 222. https://doi.org/10.1186/2193-1801-2-222

Drake, C. (1993). Effects of misspecification of the propensity score on estimators of treatment effect. Biometrics, 49(4), 1231-1236. http://www.jstor.org/stable/2532266

Elwert, F., \& Winship, C. (2014). Endogenous selection bias: The problem of conditioning on a collider variable. Annual Review of Sociology, 40(1), 31-53. https://doi.org/10.1146/annurev-soc-071913-043455

Fisher, A., Rudin, C., \& Dominici, F. (2019). All models are wrong, but many are useful: Learning a variable's importance by studying an entire class of prediction models 
simultaneously. Journal of Machine Learning Research, 20(177), 1-81. http://jmlr.org/papers/v20/18-760.html

Fong, C., Ratkovic, M., \& Imai, K. (2021). CBPS: Covariate balancing propensity score [R package version 0.22]. https://CRAN.R-project.org/package=CBPS

Fox, J., \& Monette, G. (1992). Generalized collinearity diagnostics. Journal of the American Statistical Association, 87(417), 178-183. Retrieved October 17, 2022, from http://www.jstor.org/stable/2290467

Friedman, J., Hastie, T., \& Tibshirani, R. (2010). Regularization paths for generalized linear models via coordinate descent. Journal of Statistical Software, 33(1), 1-22. http://www.jstatsoft.org/v33/i01/

Fuentes, A., Lüdtke, O., \& Robitzsch, A. (2021). Causal inference with multilevel data: A comparison of different propensity score weighting approaches [PMID: 34128730]. Multivariate Behavioral Research, 0(0), 1-24. https://doi.org/10.1080/00273171.2021.1925521

Gische, C., West, S. G., \& Voelkle, M. C. (2021). Forecasting causal effects of interventions versus predicting future outcomes. Structural Equation Modeling: A Multidisciplinary Journal, 28(3), 475-492. https://doi.org/10.1080/10705511.2020.1780598

Glynn, A. N., \& Quinn, K. M. (2010). An introduction to the augmented inverse propensity weighted estimator. Political analysis, 18(1), 36-56. https://doi.org/10.1093/pan/mpp036

Greenland, S. (2008). Invited commentary: Variable selection versus shrinkage in the control of multiple confounders. American Journal of Epidemiology, 167(5), $523-529$.

Greenland, S., Daniel, R., \& Pearce, N. (2016). Outcome modelling strategies in epidemiology: Traditional methods and basic alternatives. International Journal of Epidemiology, 45(2), 565-575. https://doi.org/10.1093/ije/dyw040 
Greifer, N. (2021). Cobalt: Covariate balance tables and plots [R package version 4.3.1]. https://CRAN.R-project.org/package=cobalt

Hansen, B. B. (2004). Full matching in an observational study of coaching for the sat. Journal of the American Statistical Association, 99(467), 609-618.

Hirano, K., \& Imbens, G. W. (2004). The propensity score with continuous treatments. In A. Gelman \& M. Xiao-Li (Eds.), Applied bayesian modeling and causal inference from incomplete-data perspectives (pp. 73-84). John Wiley \& Sons, Ltd. https://doi.org/https://doi.org/10.1002/0470090456.ch7

Hoaglin, D. C. (2016). Misunderstandings about Q and 'Cochran's Q test' in meta-analysis. Statistics in Medicine, 35(4), 485-495. https://doi.org/10.1002/sim.6632

Holland, P. W. (1986). Statistics and causal inference. Journal of the American Statistical Association, 81 (396), 945-960.

Hong, G., Yang, F., \& Qin, X. (2021). Did you conduct a sensitivity analysis? A new weighting-based approach for evaluations of the average treatment effect for the treated. Journal of the Royal Statistical Society: Series A (Statistics in Society), 184(1), 227-254. https://doi.org/https://doi.org/10.1111/rssa.12621

Huang, P.-H. (2020a). lslx: Semi-confirmatory structural equation modeling via penalized likelihood. Journal of Statistical Software, 93(7), 1-37. https://doi.org/10.18637/jss.v093.i07

Huang, P.-H. (2020b). Postselection inference in structural equation modeling [PMID: 31304796]. Multivariate Behavioral Research, 55(3), 344-360. https://doi.org/10.1080/00273171.2019.1634996

Huang, P.-H., Chen, H., \& Weng, L.-J. (2017). A penalized likelihood method for structural equation modeling. Psychometrika, 82(2), 329-354. https://doi.org/10.1007/s11336-017-9566-9 
Imai, K., \& Ratkovic, M. (2014). Covariate balancing propensity score. Journal of the Royal Statistical Society: Series B (Statistical Methodology), 76(1), 243-263. https://doi.org/https://doi.org/10.1111/rssb.12027

Ioannidis, J. P. A. (2005). Why most published research findings are false. PLOS Medicine, 2(8), e124. https://doi.org/10.1371/journal.pmed.0020124

Ioannidis, J. P. A. (2007). Why most published research findings are false: Author's reply to Goodman and Greenland. PLOS Medicine, 4 (6), 1-2. https://doi.org/10.1371/journal.pmed.0040215

Jacobucci, R., Grimm, K. J., Brandmaier, A. M., Serang, S., Kievit, R. A., Scharf, F., Li, X., \& Ye, A. (2020). regsem: Regularized structural equation modeling [R package version 1.6.2]. https://CRAN.R-project.org/package=regsem

Jacobucci, R., Grimm, K. J., \& McArdle, J. J. (2016). Regularized structural equation modeling. Structural Equation Modeling: A Multidisciplinary Journal, 23(4), 555-566. https://doi.org/10.1080/10705511.2016.1154793

Ju, C., Benkeser, D., \& van Der Laan, M. J. (2020). Robust inference on the average treatment effect using the outcome highly adaptive lasso. Biometrics, 76(1), 109-118. https://doi.org/10.1111/biom.13121

Kahan, B. C., Jairath, V., Doré, C. J., \& Morris, T. P. (2014). The risks and rewards of covariate adjustment in randomized trials: An assessment of 12 outcomes from 8 studies. Trials, 15(1), 139. https://doi.org/10.1186/1745-6215-15-139

Kainz, K., Greifer, N., Givens, A., Swietek, K., Lombardi, B. M., Zietz, S., \& Kohn, J. L. (2017). Improving causal inference: Recommendations for covariate selection and balance in propensity score methods. Journal of the Society for Social Work and Research, 8(2), 279-303. https://doi.org/10.1086/691464

Kang, J. D., \& Schafer, J. L. (2007). Demystifying double robustness: A comparison of alternative strategies for estimating a population mean from incomplete data. Statistical science, 22(4), 523-539. 
Keele, L., \& Small, D. S. (2021). Comparing covariate prioritization via matching to machine learning methods for causal inference using five empirical applications. The American Statistician, 75(4), 355-363. https://doi.org/10.1080/00031305.2020.1867638 doi: 10.1080/00031305.2020.1867638

Kelcey, B. (2011). Covariate selection in propensity scores using outcome proxies. Multivariate Behavioral Research, 46(3), 453-476. https://doi.org/10.1080/00273171.2011.570164

Koch, B., Vock, D. M., \& Wolfson, J. (2018). Covariate selection with group lasso and doubly robust estimation of causal effects. Biometrics, $74(1), 8-17$. https://doi.org/https://doi.org/10.1111/biom.12736

Kurz, C. F. (2022). Augmented inverse probability weighting and the double robustness property. Medical Decision Making, 42(2), 156-167. https://doi.org/10.1177/0272989X211027181

Lee, T., \& Shi, D. (2021). A comparison of full information maximum likelihood and multiple imputation in structural equation modeling with missing data. Psychological Methods, 26(4), 466-485. https://doi.org/10.1037/met0000381

Little, R. J., An, H., Johanns, J., \& Giordani, B. (2000). A comparison of subset selection and analysis of covariance for the adjustment of confounders. Psychological Methods, 5(4), 459-476. https://doi.org/10.1037/1082-989X.5.4.459

Liu, W., Kuramoto, S. J., \& Stuart, E. A. (2013). An introduction to sensitivity analysis for unobserved confounding in nonexperimental prevention research. Prevention Science, $14(6), 570-580$.

Loh, W. W., \& Vansteelandt, S. (2021a). Confounder selection strategies targeting stable treatment effect estimators. Statistics in Medicine, 40(3), 607-630. https://doi.org/https://doi.org/10.1002/sim.8792 
Loh, W. W., \& Vansteelandt, S. (2021b). Sensitivity analysis for unmeasured confounding via effect extrapolation. arXiv preprint arXiv:2102.01935.

Lunceford, J. K., \& Davidian, M. (2004). Stratification and weighting via the propensity score in estimation of causal treatment effects: A comparative study. Statistics in Medicine, 23(19), 2937-2960. https://doi.org/10.1002/sim.1903

Maldonado, G., \& Greenland, S. (1993). Simulation study of confounder-selection strategies. American Journal of Epidemiology, 138(11), 923-936.

McCaffrey, D. F., Ridgeway, G., \& Morral, A. R. (2004). Propensity score estimation with boosted regression for evaluating causal effects in observational studies. Psychological Methods, 9(4), 403-425. https://doi.org/10.1037/1082-989X.9.4.403

Mickey, R. M., \& Greenland, S. (1989). The impact of confounder selection criteria on effect estimation. American Journal of Epidemiology, 129(1), 125-137. https://doi.org/10.1093/oxfordjournals.aje.a115101

Parast, L., \& Griffin, B. A. (2020). Quantifying the bias due to observed individual confounders in causal treatment effect estimates. Statistics in Medicine, 39(18), 2447-2476. https://doi.org/https://doi.org/10.1002/sim.8549

Pearl, J. (2009). Causality: Models, reasoning and inference (2nd). Cambridge University Press. https://doi.org/10.1017/CBO9780511803161

Pearl, J. (2011). Invited Commentary: Understanding Bias Amplification. American Journal of Epidemiology, 174(11), 1223-1227. https://doi.org/10.1093/aje/kwr352

Powell, M. G., Hull, D. M., \& Beaujean, A. A. (2020). Propensity score matching for education data: Worked examples. The Journal of Experimental Education, 88(1), 145-164. https://doi.org/10.1080/00220973.2018.1541850

Pregibon, D. (1982). Resistant fits for some commonly used logistic models with medical applications. Biometrics, 485-498. https://doi.org/10.2307/2530463

R Core Team. (2021). R: A language and environment for statistical computing. $\mathrm{R}$ Foundation for Statistical Computing. Vienna, Austria. https://www.R-project.org/ 
Ren, D., Stavrova, O., \& Loh, W. W. (2022). Nonlinear effect of social interaction quantity on psychological well-being: Diminishing returns or inverted u? Journal of Personality and Social Psychology, 122, 1056-1074. https://doi.org/10.1037/pspi0000373

Robins, J. M., Rotnitzky, A., \& Zhao, L. P. (1994). Estimation of regression coefficients when some regressors are not always observed. Journal of the American Statistical Association, 89(427), 846-866.

Rosenbaum, P. R. (1987a). Sensitivity analysis for certain permutation inferences in matched observational studies. Biometrika, 74, 13-26.

Rosenbaum, P. R. (1984). From association to causation in observational studies: The role of tests of strongly ignorable treatment assignment. Journal of the American Statistical Association, $79(385), 41-48$. https://doi.org/10.1080/01621459.1984.10477060

Rosenbaum, P. R. (1987b). Model-based direct adjustment. Journal of the American Statistical Association, 82(398), 387-394.

Rosenbaum, P. R. (2002). Observational Studies. New York : Springer Includes bibliographical references and index.

Rosenbaum, P. R., \& Rubin, D. B. (1983). The central role of the propensity score in observational studies for causal effects. Biometrika, 70(1), 41-55. https://doi.org/10.1093/biomet/70.1.41

Rubin, D. B. (1986). Comment: Which ifs have causal answers. Journal of the American Statistical Association, 81 (396), 961-962. https://doi.org/10.1080/01621459.1986.10478355

Rubin, D. B. (1990). [On the Application of Probability Theory to Agricultural Experiments. Essay on Principles. Section 9.] Comment: Neyman (1923) and Causal Inference in Experiments and Observational Studies. Statistical Science, 5(4), 472-480. https://doi.org/10.1214/ss/1177012032 
Rubin, D. B. (2007). The design versus the analysis of observational studies for causal effects: Parallels with the design of randomized trials. Statistics in Medicine, 26(1), 20-36. https://doi.org/10.1002/sim.2739

Sclove, S. L. (1987). Application of model-selection criteria to some problems in multivariate analysis. Psychometrika, 52(3), 333-343. https://doi.org/10.1007/BF02294360

Sengewald, M.-A., \& Mayer, A. (2022). Causal effect analysis in nonrandomized data with latent variables and categorical indicators: The implementation and benefits of effectliter. Psychological Methods, Advance online publication. https://doi.org/10.1037/met0000489

Sengewald, M.-A., Steiner, P. M., \& Pohl, S. (2019). When does measurement error in covariates impact causal effect estimates? Analytic derivations of different scenarios and an empirical illustration. British Journal of Mathematical and Statistical Psychology, 72(2), 244-270. https://doi.org/https://doi.org/10.1111/bmsp.12146

Setodji, C. M., McCaffrey, D. F., Burgette, L. F., Almirall, D., \& Griffin, B. A. (2017). The right tool for the job: Choosing between covariate-balancing and generalized boosted model propensity scores. Epidemiology, 28(6), 802-811. https://doi.org/10.1097/EDE.0000000000000734

Shadish, W. R., \& Steiner, P. M. (2010). A primer on propensity score analysis [Quantitative Research Methodology]. Newborn and Infant Nursing Reviews, 10(1), 19-26. https://doi.org/https://doi.org/10.1053/j.nainr.2009.12.010

Shortreed, S. M., \& Ertefaie, A. (2017). Outcome-adaptive lasso: Variable selection for causal inference. Biometrics, 73(4), 1111-1122. https://doi.org/10.1111/biom.12679 Splawa-Neyman, J., Dabrowska, D. M., \& Speed, T. P. (1990). On the Application of Probability Theory to Agricultural Experiments. Essay on Principles. Section 9. Statistical Science, 5(4), 465-472. https://doi.org/10.1214/ss/1177012031 
Steiner, P. M., Cook, T. D., Li, W., \& Clark, M. H. (2015). Bias reduction in quasi-experiments with little selection theory but many covariates. Journal of Research on Educational Effectiveness, 8(4), 552-576. https://doi.org/10.1080/19345747.2014.978058

Steiner, P. M., Cook, T. D., Shadish, W. R., \& Clark, M. H. (2010). The importance of covariate selection in controlling for selection bias in observational studies. Psychological Methods, 15(3), 250-267. https://doi.org/10.1037/a0018719

Stuart, E. A. (2010). Matching methods for causal inference: A review and a look forward. Statistical Science, 25(1), 1-21. https://doi.org/10.1214/09-STS313

Stuart, E. A., \& Rubin, D. B. (2008). Best practices in quantitative methods. In J. Osborne (Ed.). SAGE Publications, Inc. https://doi.org/10.4135/9781412995627

Thoemmes, F. J. (2015). M-bias, butterfly bias, and butterfly bias with correlated causes a comment on Ding and Miratrix (2015). Journal of Causal Inference, 3(2), 253-258. https://doi.org/doi:10.1515/jci-2015-0012

Thoemmes, F. J., \& West, S. G. (2011). The use of propensity scores for nonrandomized designs with clustered data. Multivariate Behavioral Research, 46(3), 514-543. https://doi.org/10.1080/00273171.2011.569395

Tibshirani, R. (2011). Regression shrinkage and selection via the LASSO: A retrospective. Journal of the Royal Statistical Society: Series B (Statistical Methodology), 73(3), 273-282. https://doi.org/10.1111/j.1467-9868.2011.00771.x

Tsiatis, A. A., Davidian, M., Zhang, M., \& Lu, X. (2008). Covariate adjustment for two-sample treatment comparisons in randomized clinical trials: A principled yet flexible approach. Statistics in Medicine, 27(23), 4658-4677. https://doi.org/https://doi.org/10.1002/sim.3113

Tutz, G., \& Gertheiss, J. (2016). Regularized regression for categorical data. Statistical Modelling, 16(3), 161-200. https://doi.org/10.1177/1471082X16642560 
van Breukelen, G. J. P. (2013). ANCOVA versus CHANGE from baseline in nonrandomized studies: The difference. Multivariate Behavioral Research, 48(6), 895-922. https://doi.org/10.1080/00273171.2013.831743

VanderWeele, T. J. (2019). Principles of confounder selection. European Journal of Epidemiology, 34(3), 211-219. https://doi.org/10.1007/s10654-019-00494-6

Vansteelandt, S., Bekaert, M., \& Claeskens, G. (2012). On model selection and model misspecification in causal inference. Statistical Methods in Medical Research, 21(1), $7-30$.

Vansteelandt, S., \& Daniel, R. M. (2014). On regression adjustment for the propensity score. Statistics in Medicine, 33(23), 4053-4072. https://doi.org/10.1002/sim.6207

Wang, C., Dominici, F., Parmigiani, G., \& Zigler, C. M. (2015). Accounting for uncertainty in confounder and effect modifier selection when estimating average causal effects in generalized linear models. Biometrics, $71(3), 654-665$. https://doi.org/10.1111/biom.12315

Westreich, D., Cole, S. R., Funk, M. J., Brookhart, M. A., \& Stürmer, T. (2011). The role of the c-statistic in variable selection for propensity score models. Pharmacoepidemiology and Drug Safety, 20(3), 317-320. https://doi.org/https://doi.org/10.1002/pds.2074

Williamson, B. D., Gilbert, P. B., Carone, M., \& Simon, N. (2021). Nonparametric variable importance assessment using machine learning techniques. Biometrics, 77(1), 9-22. https://doi.org/https://doi.org/10.1111/biom.13392

Witte, J., \& Didelez, V. (2019). Covariate selection strategies for causal inference: Classification and comparison. Biometrical Journal, 61(5), 1270-1289. https://doi.org/10.1002/bimj.201700294

Wooldridge, J. M. (2016). Should instrumental variables be used as matching variables? Research in Economics, 70(2), 232-237. https://doi.org/https://doi.org/10.1016/j.rie.2016.01.001 
Yuan, M., \& Lin, Y. (2006). Model selection and estimation in regression with grouped variables. Journal of the Royal Statistical Society: Series B (Statistical Methodology), 68(1), 49-67.

Zhong, Y., Kennedy, E. H., Bodnar, L. M., \& Naimi, A. I. (2021). AIPW: An R package for augmented inverse probability weighted estimation of average causal effects. American Journal of Epidemiology. https://doi.org/10.1093/aje/kwab207

Zurovaca, J., Cook, T. D., Deke, J., Finucane, M. M., Chaplin, D., Coopersmith, J. S., Barna, M., \& Forrow, L. V. (2021). Absolute and relative bias in eight common observational study designs: Evidence from a meta-analysis. arXiv preprint $\operatorname{arXiv:2111.06941.~}$ 


\section{Appendix A}

\section{Diagnostic for numerically assessing the relative stability of the effect estimators}

In this section, we describe a diagnostic that numerically assesses the relative stability or (in)sensitivity of the difference $\widehat{\psi}_{j}-\widehat{\psi}_{J}$, while taking into account its variability, that can directly optimize the quality of the treatment effect estimator. We will use an inverse variance weighted average of the differences $\widehat{\psi}_{j}-\widehat{\psi}_{J}$ within a (moving) window of consecutive subsets. For simplicity, we will use (symmetric) windows of width three centered around each subset $j=2, \ldots, J-1$. The diagnostic for the $j$-th subset is therefore defined as:

$$
Q_{j}=\sum_{k=j-1}^{j+1} w_{k}\left\{\left(\widehat{\psi}_{k}-\widehat{\psi}_{J}\right)-\overline{\bar{\psi}_{j}}\right\}^{2}
$$

where the weights $w_{k}$ and weighted average $\overline{\widehat{\psi}_{j}}$ are respectively defined as:

$$
w_{k}=\left\{\operatorname{var}\left(\widehat{\psi}_{k}-\widehat{\psi}_{J}\right)\right\}^{-1}, \quad \overline{\widehat{\psi}_{j}}=\left(\sum_{k=j-1}^{j+1} w_{k}\right)^{-1} \sum_{k=j-1}^{j+1} w_{k}\left(\widehat{\psi}_{k}-\widehat{\psi}_{J}\right) .
$$

It is hence assumed that $w_{J}=0$. Notwithstanding the $\widehat{\psi}_{J}$ estimators canceling each other out within the quadratic term in A1, we use weights that are inversely proportional to the variance of the approximate biases encoded by the differences $\widehat{\psi}_{j}-\widehat{\psi}_{J}$ toward attaining unbiased inference, rather than the variance of $\widehat{\psi}_{j}$ alone which focuses on the efficiency of the effect estimator. Therefore, we will define the smallest subset with the most stable (standardized) difference to minimize the Q statistic; i.e.,

$$
\min _{j=2, \ldots, J-1} Q_{j}
$$

Readers familiar with the meta-analysis literature will recognize that the weighted average defined in (A1) adopts the same form as "Cochran's Q statistic" (Hoaglin, 2016) for assessing heterogeneity of effect-size estimates from separate studies. Because the Q statistic is used merely to summarize the (in)stability of the effect estimators across different subsets, and not compared against its asymptotic distribution for inference, no assumption of independence between (non-)consecutive subsets is needed. 


\section{Appendix B}

\section{Doubly-robust augmented inverse propensity weighted estimators}

In this section, we provide closed-form expressions for the difference between doubly-robust augmented inverse propensity (score), or inverse probability (of treatment), weighted estimators from two subsets, as well as their (asymptotic) variance estimators (1), under (non-)linear parametric regression models for the treatment and the outcome. For a binary treatment, denote the (logistic) treatment model, conditional on the selected covariates in the $j$-th subset $L^{j}$, by:

$$
p\left(L_{i}^{j}\right)=\mathrm{E}\left(A_{i} \mid L_{i}^{j}\right)=\operatorname{Pr}\left(A_{i}=1 \mid L_{i}^{j}\right)=\operatorname{expit}\left(\alpha_{j} L_{i}^{j}\right)
$$

where the subscript $i$ of $L_{i}^{j}$ denotes individual $i$ and $\operatorname{expit}(x)=\exp (x) /\{1+\exp (x)\}$. Define the inverse probability of treatment weight (Lunceford \& Davidian, 2004 . Rosenbaum, 1987b) for individual $i$ as:

$$
W_{i}^{j}=\frac{A_{i}}{p\left(L_{i}^{j}\right)}+\frac{1-A_{i}}{1-p\left(L_{i}^{j}\right)} .
$$

The weight $W_{i}^{j}$ is the reciprocal of the conditional probability of individual $i$ being assigned their observed treatment $A_{i}$ given their covariates $L_{i}^{j}$. Let $\widehat{W}_{i}^{j}$ and $\widehat{p}\left(L_{i}^{j}\right)$ denote the estimated weights and treatment probabilities, respectively, obtained by substituting estimators for $\alpha_{j}$ (using e.g., maximum likelihood) in the treatment model. Fit the outcome regression model $\mathrm{E}\left(Y_{i} \mid A_{i}, L_{i}^{j}\right)=h^{-1}\left(\psi_{j}^{*} A_{i}+\beta_{j} L_{i}^{j}\right)$ to the observed data, where $h^{-1}$ is the inverse of the link function $h$. (The $*$ superscript indicates a conditional effect that may differ from the marginal effect $\psi_{j}$.) Let $\widehat{\mathrm{E}}\left(Y_{i} \mid A_{i}, L_{i}^{j}\right)$ denote the fitted outcome model obtained by plugging in estimators (using e.g., maximum likelihood) for $\psi_{j}^{*}$ and $\beta_{j}$. The estimator of the marginal treatment effect $\psi=\mathrm{E}\left(Y^{1}\right)-\mathrm{E}\left(Y^{0}\right)$ in the $j$-th subset is:

$$
\begin{aligned}
\widehat{\psi}_{j}= & n^{-1} \sum_{i} \frac{A_{i}}{\widehat{p}\left(L_{i}^{j}\right)}\left\{Y_{i}-\widehat{\mathrm{E}}\left(Y_{i} \mid A_{i}=1, L_{i}^{j}\right)\right\}-\frac{1-A_{i}}{1-\widehat{p}\left(L_{i}^{j}\right)}\left\{Y_{i}-\widehat{\mathrm{E}}\left(Y_{i} \mid A_{i}=0, L_{i}^{j}\right)\right\} \\
& +\left\{\widehat{\mathrm{E}}\left(Y_{i} \mid A_{i}=1, L_{i}^{j}\right)-\widehat{\mathrm{E}}\left(Y_{i} \mid A_{i}=0, L_{i}^{j}\right)\right\} .
\end{aligned}
$$


An asymptotic expansion around $\psi$ yields the so-called "influence function" for individual $i$ as:

$$
\begin{aligned}
\phi_{i}^{j}= & \frac{A_{i}}{\widehat{p}\left(L_{i}^{j}\right)}\left\{Y_{i}-\widehat{\mathrm{E}}\left(Y_{i} \mid A_{i}=1, L_{i}^{j}\right)\right\}-\frac{1-A_{i}}{1-\widehat{p}\left(L_{i}^{j}\right)}\left\{Y_{i}-\widehat{\mathrm{E}}\left(Y_{i} \mid A_{i}=0, L_{i}^{j}\right)\right\} \\
& +\left\{\widehat{\mathrm{E}}\left(Y_{i} \mid A_{i}=1, L_{i}^{j}\right)-\widehat{\mathrm{E}}\left(Y_{i} \mid A_{i}=0, L_{i}^{j}\right)\right\}-\psi .
\end{aligned}
$$

Let $\widehat{\phi}_{i}^{j}$ denote the estimated influence function, obtained by plugging in coefficient estimates in the treatment and outcome models, and substituting the unknown value of $\psi$ with the estimator from the $j$-th subset $\widehat{\psi}_{j}$. When both treatment and outcome models are correctly specified, the variance of the difference between effect estimators from two subsets, e.g., $j$ and $k$, is consistently estimated by $1 / n$ times the sample variance (denoted by $\widehat{\operatorname{var}})$ of the corresponding difference in estimated influence functions:

$$
\widehat{\operatorname{var}}\left(\widehat{\psi}_{j}-\widehat{\psi}_{k}\right)=\{n(n-1)\}^{-1} \sum_{i}\left(\widehat{\phi}_{i}^{j}-\widehat{\phi}_{i}^{k}\right)^{2}
$$

Consistency and asymptotic normality of the standardized difference (1) with zero mean and unit variance directly follow from the law of large numbers and the central limit theorem. 